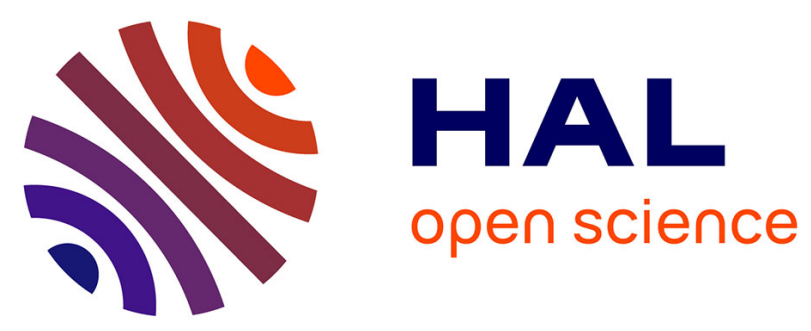

\title{
Alfvén vortex filaments observed in magnetosheath downstream of a quasi-perpendicular bow shock.
}

Olga Alexandrova, Anne Mangeney, M. Maksimovic, N. Cornilleau-Wehrlin, J.-M. Bosqued, M. André

\section{- To cite this version:}

Olga Alexandrova, Anne Mangeney, M. Maksimovic, N. Cornilleau-Wehrlin, J.-M. Bosqued, et al.. Alfvén vortex filaments observed in magnetosheath downstream of a quasi-perpendicular bow shock.. Journal of Geophysical Research Space Physics, 2006, 111 (A12), pp.A12208. 10.1029/2006JA011934 . hal-00153549

\author{
HAL Id: hal-00153549 \\ https://hal.science/hal-00153549
}

Submitted on 14 Feb 2016

HAL is a multi-disciplinary open access archive for the deposit and dissemination of scientific research documents, whether they are published or not. The documents may come from teaching and research institutions in France or abroad, or from public or private research centers.
L'archive ouverte pluridisciplinaire HAL, est destinée au dépôt et à la diffusion de documents scientifiques de niveau recherche, publiés ou non, émanant des établissements d'enseignement et de recherche français ou étrangers, des laboratoires publics ou privés. 


\title{
Alfvén vortex filaments observed in magnetosheath downstream of a quasi-perpendicular bow shock
}

\author{
O. Alexandrova, ${ }^{1}$ A. Mangeney, ${ }^{1}$ M. Maksimovic, ${ }^{1}$ N. Cornilleau-Wehrlin, ${ }^{2}$ \\ J.-M. Bosqued, ${ }^{3}$ and M. André ${ }^{4}$ \\ Received 23 June 2006; revised 25 August 2006; accepted 31 August 2006; published 15 December 2006.
}

[1] Magnetic field fluctuations in the frequency range [0.02-12.5] $\mathrm{Hz}$ are studied with the four Cluster satellites in the Earth magnetosheath downstream of a quasi-perpendicular bow shock. The turbulent spectrum presents a spectral break accompanied by a broad maximum usually interpreted as due to Alfvén ion cyclotron waves. In this paper we establish that this spectral knee corresponds to space-localized coherent magnetic structures in the form of Alfvén vortices. The Alfvén vortex is a nonlinear cylindrical Alfvén wave, quasi-parallel to the mean magnetic field $\mathbf{B}_{0}$ and propagating in a plane perpendicular to $\mathbf{B}_{0}$. In this plane the observed vortices are localized within $20 c / \omega_{p i}$. The frequent observations of such structures indicate their stability in the plasma. Therefore the Alfvén vortices can be an important element in the magnetosheath turbulence. The possible origins of these vortices, such as a strong turbulence or the filamentation instability of an Alfvén wave, are discussed.

Citation: Alexandrova, O., A. Mangeney, M. Maksimovic, N. Cornilleau-Wehrlin, J.-M. Bosqued, and M. André (2006), Alfvén vortex filaments observed in magnetosheath downstream of a quasi-perpendicular bow shock, J. Geophys. Res., 111, A12208, doi:10.1029/2006JA011934.

\section{Introduction}

[2] The Earth magnetosheath is the collisionless plasma found between the Earth bow shock and the magnetosphere boundary (the magnetopause). In this region the solar wind slows down and heats up. A notable feature of the magnetosheath is the ion temperature anisotropy: the temperature perpendicular to the mean magnetic field $\left(T_{i \perp}\right)$ is larger than the parallel one $\left(T_{i \|}\right)$. This anisotropy is a source of free energy and its liberation proceeds through the generation of waves. The linear Vlasov theory for homogeneous plasmas predicts that if the plasma beta, the ratio between the plasma pressure $p$ and the magnetic pressure, is small $\left(\beta=2 \mu_{0} p / B^{2} \leq 1\right)$, Alfvén ion cyclotron (AIC) waves will grow, while for a high beta $(\beta \geq 5)$ mirror waves will grow [Schwartz et al., 1996]. These waves then scatter and diffuse the particles, reducing the anisotropy. Most of previous works on the magnetosheath were devoted to the identification of AIC and mirror waves [Lacombe et al., 1992; Anderson et al., 1994; Lucek et al., 2001; Sahraoui et al., 2003; Alexandrova et al., 2004] to confirm this scenario of anisotropy relaxation. Very convincing results have been

\footnotetext{
${ }^{1}$ Observatoire de Paris-Meudon, Laboratoire d'Etudes Spatiales et d'Instrumentation en Astrophysique, Centre National de la Recherche Scientifique, Meudon, France.

${ }^{2}$ Centre d'Etude des Environnements Terrestre et Plantaires, L'Institut Pierre-Simon Laplace, Vélizy, France.

${ }^{3}$ Centre d'Etude Spatiale des Rayonnements, Centre National de la Recherche Scientifique, Toulouse, France.

${ }^{4}$ Swedish Institute of Space Physics, Umeiå University, Umeiå, Sweden.

Copyright 2006 by the American Geophysical Union. 0148-0227/06/2006JA011934
}

obtained and discussed by Lacombe and Belmont [1995], where one can see that AIC and Mirror instabilities control the magnetosheath state. That was confirmed by numerical simulations such as those described by Hellinger et al. [2003].

[3] The linear theory of plasma instabilities, nevertheless, cannot explain the observed magnetic spectra which extend over a large number of frequency decades [Rezeau et al., 1999; Czaykowska et al., 2001]. It is commonly accepted that the magnetosheath is turbulent and this turbulence is weak, i.e., an incoherent mixture of almost linear wave modes [Rezeau et al., 1999]. In a case study in the magnetosheath near the magnetopause, it was shown that the turbulent spectrum in the frequency range above the proton cyclotron frequency, $f>f_{c p}=e B / m_{p}$, is formed by zero frequency mirror waves convected by the plasma flow across the spacecraft [Sahraoui et al., 2006].

[4] A statistical study by Czaykowska et al. [2001] has shown that the power spectrum of magnetic fluctuations in a large frequency range around the proton cyclotron frequency can be described by two power laws with a break, i.e., a change of slope, in the vicinity of $f_{c p}$. For a low beta in the solar wind $(\beta<0.5)$, this spectral break in the magnetosheath is accompanied by a "knee" (a local and broad maximum), which was interpreted as the signature of AIC waves. This interpretation is in agreement with the quasilinear scenario of the temperature anisotropy relaxation. However, the AIC instability is usually visible in the spectrum as a sharp peak and not as a large maximum [see, e.g., Alexandrova et al., 2004]. In the solar wind turbulence the spectral break has been also observed, but not the knee [see, e.g., Leamon et al., 1998]. 
[5] In this paper we will study in detail the nature of the turbulent fluctuations responsible for the spectral knee described above. For that purpose we analyze magnetic field fluctuations measured by the Cluster satellites on 31 March 2001 in the middle of the magnetosheath behind a quasi-perpendicular bow shock.

[6] We perform both a one satellite and multisatellite analysis on the magnetic fluctuations corresponding to this knee. A single satellite analysis shows that the magnetic fluctuations are Alfvénic; if they are assumed to be a superposition of plane waves, a minimum variance analysis suggests that the wave vector is nearly parallel to the unperturbed magnetic filed $\mathbf{B}_{0}$. Instead, the multisatellite analysis shows that these fluctuations are localized in the plane perpendicular to $\mathbf{B}_{0}$, in contrast to the hypothesis of parallel propagating Alfvén plane waves. These localized Alfvénic structures have the form of field-aligned filaments, with velocities mainly perpendicular to the mean field and with transverse dimensions of the order of 20 ion inertial length $c / \omega_{p i}\left(\omega_{p i}=\sqrt{e^{2} n /\left(\varepsilon_{0} m_{p}\right)}\right.$ being the proton plasma frequency). The frequent observations of such filaments in the magnetosheath suggests that they are stable structures in the plasma with a long life time.

[7] A good model for these localized Alfvénic structures is known under the name of Alfvén vortex filaments [Petviashvili and Pokhotelov, 1992] which are cylindrical analogues of the nonlinear Alfvén wave. In the simplest case, they are solutions of the incompressible ideal magnetohydrodynamic (MHD) equations (see Kadomtsev and Pogutse [1974] and Strauss [1976] for the original references). The axis of the vortex is quasi-parallel to $\mathbf{B}_{0}$. The qualitative difference with the Alfvén wave is that the vortex propagates in a plane perpendicular to the unperturbed magnetic field with a velocity determined by the projection of this field $\mathbf{B}_{0}$ on the plane perpendicular to the vortex axis. In the case when the vortex axis is strictly parallel to the field, this velocity is equal to zero. Moreover, the well-known relation $\delta V_{\perp} / V_{A}= \pm \delta B_{\perp} / B_{0}\left(V_{A}=B / \sqrt{\mu_{0} \rho}\right.$ being the Alfvén speed) between the perpendicular magnetic field and velocity fluctuations in the Alfvén wave is replaced for the vortex by a more general relation $\delta V_{\perp} / V_{A}=$ $\xi \delta B_{\perp} / B_{0}$, where $\xi$ is determined by the vortex velocity.

[8] To the authors' knowledge, this is the first clear identification of such an Alfvén vortex in a space plasma. We discuss some possible generating mechanisms in the last section of this paper.

\section{Data and Observations}

[9] In this paper we study one of the magnetosheath crossings on 31 March 2001 by the Cluster mission. Three magnetic components are obtained from the mixing [Alexandrova et al., 2004] of the waveforms measured by the search coils of the STAFF experiment at $0.04 \mathrm{~s}$ time resolution [Cornilleau-Wehrlin et al., 2003] and the smoothed data from the FGM experiment at $4 \mathrm{~s}$ resolution [Balogh et al., 2001]. Two electric components in the plane of the satellite spin, i.e., $E_{X}$ and $-E_{Y}$ in the GSE frame, are measured by the Electric Fields and Waves (EFW) experiment [Gustafsson et al., 1997] with $0.04 \mathrm{~s}$ time resolution. The CIS instrument [Rème et al., 2001] measures full three-dimensional (3-D) ion distribution functions

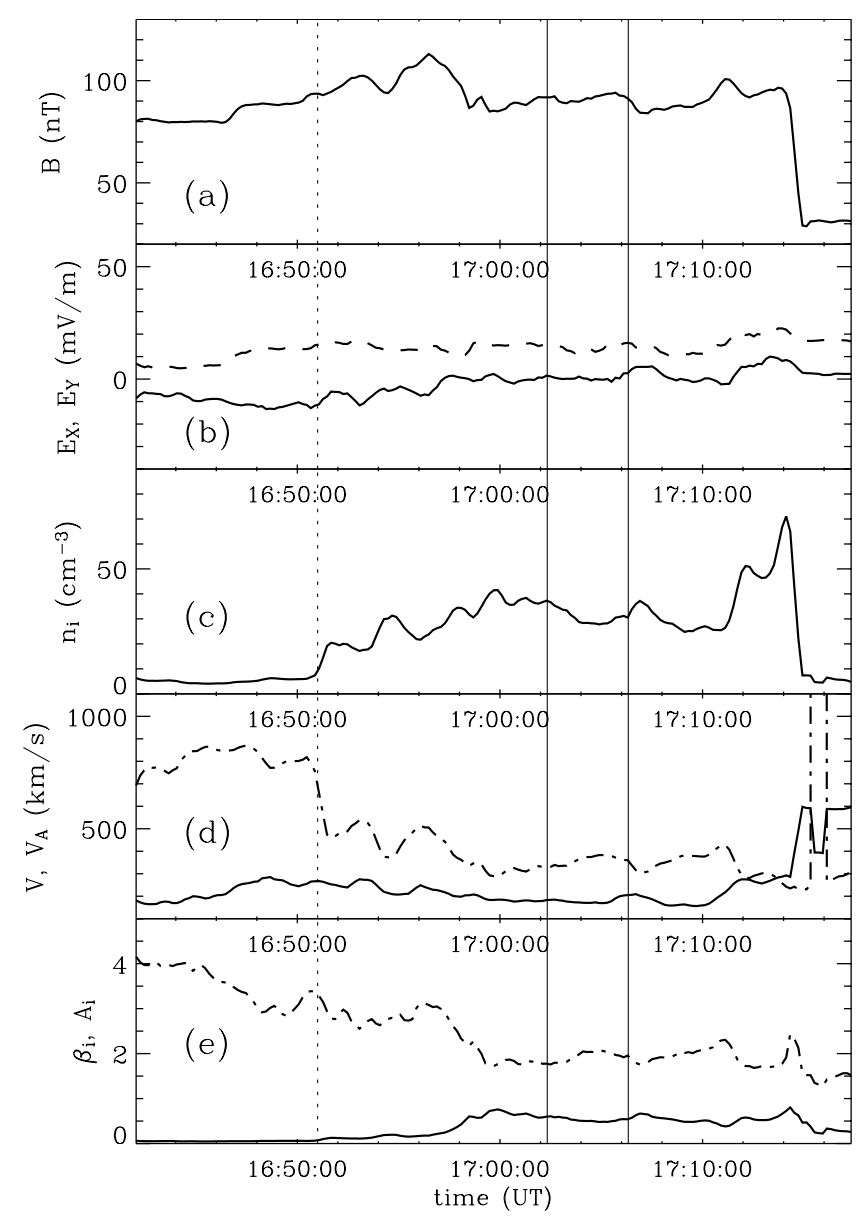

Figure 1. Cluster-3, fields, and plasma parameters during the time period 1642:00-1717:24 UT on 31 March 2001; (a) magnetic field amplitude; (b) two components of electric field in the GSE frame, $E_{X}$ (solid line) and $E_{Y}$ (dashed line); (c) CIS ions density $n_{i}$; (d) CIS plasma bulk velocity (solid line) and Alfvén velocity $V_{A}$ (dashed dotted line); (e) ion plasma beta $\beta_{i}$ (solid line) and ion temperature anisotropy $A_{i}=T_{i \perp} / T_{i \|}$ (dashed dotted line). The vertical dotted line indicates the end of the cusp region. The two vertical solid lines indicate the time period 1702:20-1706:20 UT where the magnetosheath is rather uniform.

and their moments (density, bulk velocity, and temperature) at a time resolution up to the spin period, that is $4 \mathrm{~s}$. In this paper, we use the Cluster-3 Hot Ion Analyser (HIA) data (without ion species resolution) with $12 \mathrm{~s}$ time resolution.

\subsection{Selected Interval}

[10] Figure 1 shows Cluster-3 (C3) measurements of fields and plasma parameters for the time period 1642:00-1717:24 UT. In Figure 1a, the magnetic field strength is shown. Figure $1 \mathrm{~b}$ displays two electric field components in GSE frame; $E_{Y}$ is represented by the dotted line. In Figure $1 \mathrm{c}$ the CIS(HIA) ion density $n_{i}$ is shown. In Figure $1 \mathrm{~d}$, we present the plasma bulk velocity $V$ (solid line) and the Alfvén speed $V_{A}$ (dashed-dotted line). In Figure 1e the ion beta $\left(\beta_{i}\right)$ and the ion temperature anisotropy $A_{i}=T_{i \perp} /$ $T_{i \|}$ (dashed-dotted line) are presented. The sudden change in 
the plasma density (Figure 1c) at about 1651:00 UT (vertical dotted line) corresponds to the end of the cusp region and the beginning on the magnetosheath (I. Dandouras, private communication, 2005). Cluster crosses the bow shock at 1714:40 UT.

[11] In this paper we study in details a magnetosheath period, marked out in Figure 1 by the two vertical solid lines. During this time period, 1702:20-1706:20 UT, all the Cluster satellites are in a rather uniform region of the magnetosheath, where the average magnetic field and plasma bulk velocity in GSE frame are $\mathbf{B}_{0}=(20.6$, $-11.0,-87.6) \mathrm{nT}$ and $\mathbf{V}_{0}=(-168.8,11.2,58.4) \mathrm{km} / \mathrm{s}$, respectively. Using these vectors, we construct a reference frame based on the magnetic field and plasma velocity. The unit vectors of the magnetic field and the velocity are $\mathbf{e}_{\mathbf{z}}$ and $\mathbf{e}_{\mathbf{v}}$, respectively, the unit vector $\mathbf{e}_{\mathbf{x}}=\left[\mathbf{e}_{\mathbf{z}} \times \mathbf{e}_{\mathbf{v}}\right] \times \mathbf{e}_{\mathbf{z}} /\left|\mathbf{e}_{\mathbf{z}} \times \mathbf{e}_{\mathbf{v}}\right|$ is perpendicular to $\mathbf{B}_{0}$ in the plane containing $\mathbf{B}_{0}$ and $\mathbf{V}_{0}$, $\mathbf{e}_{\mathbf{y}}=\mathbf{e}_{\mathbf{z}} \times \mathbf{e}_{\mathbf{x}}$ is chosen to complete the direct orthonormal frame $\left(\mathbf{e}_{\mathbf{x}}, \mathbf{e}_{\mathbf{y}}, \mathbf{e}_{\mathbf{z}}\right)$. All over the paper we will be working in this reference system. The mean parameters of the plasma which are used in this paper are the following: $B_{0}=90 \mathrm{nT}$, $n_{0}=30 \mathrm{~cm}^{-3}, V_{A}=360 \mathrm{~km} / \mathrm{s}, T_{i}=360 \mathrm{eV}, \beta_{i}=0.5$.

\subsection{Wavelet Spectrum}

[12] We use here the Morlet wavelet transform, which provides a good compromise between the frequency resolution, necessary to identify the spectral knee, and the time resolution, necessary to identify local structures as the Alfvén vortices. The Morlet wavelet is a wave of frequency $f$ modulated by a Gaussian:

$$
\psi_{0}(t)=\pi^{-1 / 4} \exp [-i 2 \pi f t] \exp \left[-t^{2} / 2\right]
$$

with $t$ being the time variable. The best time/frequency resolution is achieved while $(2 \pi f)=6$ [Torrence and Compo, 1998]. The wavelet transform of the $j$ th component of the magnetic field $B_{j}\left(t_{i}\right)$, a data time series with $i=0, \ldots$, $N-1$, is the convolution of $B_{j}(t)$ with a scaled and translated version of $\psi_{0}(t)$

$$
\mathcal{W}_{j}(m, t)=\sum_{i=0}^{N-1} B_{j}\left(t_{i}\right) \psi^{*}\left[\left(t_{i}-t\right) / m\right]
$$

where the asterisk indicates the complex conjugate and $m$ is a time scale (the corresponding frequency is $f=1 / \mathrm{m}$ ).

[13] For the selected magnetosheath time period (4 min, from 1702:20 to 1706:20 UT), the scalogram of Figure 2b displays the distribution of the total energy (color levels) of the magnetic field fluctuations as a function of scale and time

$$
\mathcal{W}^{2}(m, t)=\sum_{j=x, y, z}\left|\mathcal{W}_{j}(m, t)\right|^{2}
$$

The solid line indicates the "cone of influence" where the Morlet coefficients are affected by end effects [Torrence and Compo, 1998]. We introduce here the frequency $f_{\text {coi }}$ that corresponds to the scale tangent to the "cone of influence" curve. In our case this scale is around $50 \mathrm{~s}$, not visible on the scalogram.
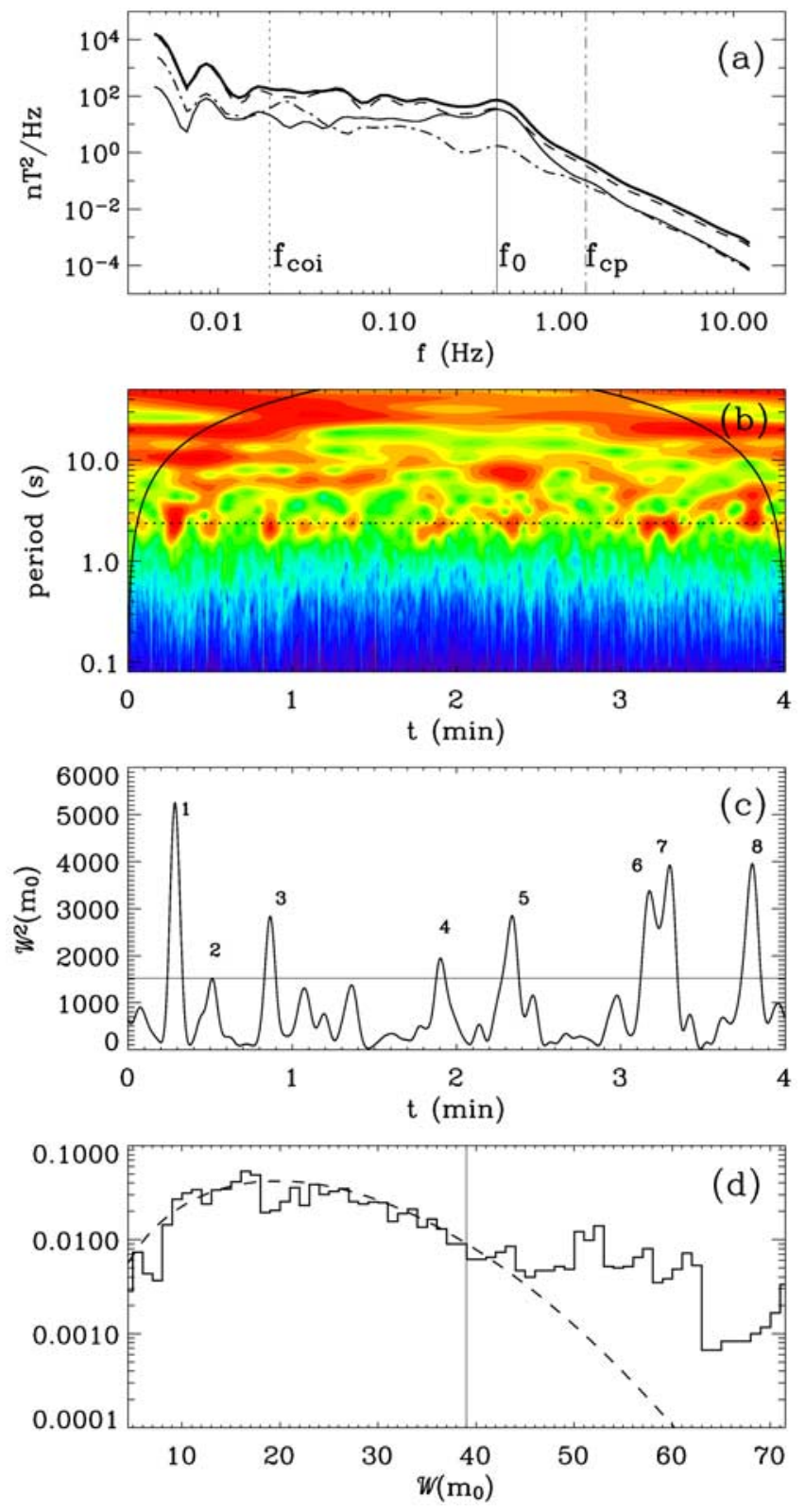

Figure 2. Spectral characteristics of magnetic field fluctuations measured during $4 \mathrm{~min}$ from 1702:20 UT, 31 March 2001: (a) Total power spectral density (thick solid line) and power spectral densities of the components $\mathcal{S}_{x}$ (solid line), $\mathcal{S}_{y}$ (dashed line), $\mathcal{S}_{z}$ (dashed-dotted line). (b) Morlet scalogram $\mathcal{W}^{2}(m, t)$, the scale $m_{0}=2.37 \mathrm{~s}$ is indicated by the dotted-line. (c) Time evolution of the magnetic energy of the scale $m_{0}, \mathcal{W}^{2}\left(m_{0}, t\right)$; the horizontal line corresponds to the threshold energy. (d) Histogram of the $\mathcal{W}\left(m_{0}, t\right)$ values, that is compared to the probability density for the amplitudes of the Gaussian field (dashed line), see equation (5); the vertical line corresponds to $\mathcal{W}\left(m_{0}\right)=39$, the threshold for the energetic peaks.

[14] Figure 2a shows the power spectral densities (PSD) of the three magnetic field components

$$
\mathcal{S}_{j}\left[\mathrm{nT}^{2} / \mathrm{Hz}\right]=\frac{2 T}{N^{2}} \sum_{t^{\prime}=0}^{N-1}\left|\mathcal{W}_{j}\left(m, t^{\prime}\right)\right|^{2}, \quad j=x, y, z
$$


and of their sum (the total PSD, solid thick line). The total PSD is in fact the result of the time integration of the scalogram of Figure $2 \mathrm{~b}$. With the data resolution $\delta t=0.04 \mathrm{~s}$, the $4 \mathrm{~min}$ data set covers a frequency range $[0.004-12.5] \mathrm{Hz}$ but the frequencies $f<f_{\text {coi }} \simeq 0.02 \mathrm{~Hz}$ (see the vertical dotted line) are affected by the end effects. The total PSD can be fitted by two power laws: $\sim f^{-\frac{1}{2}}$ for the low-frequency range $[0.02-0.3] \mathrm{Hz}$ and $\sim f^{-3}$ for the high-frequency range $[0.8-$ 12.5] Hz. The vertical solid line indicates the central frequency $f_{0}=0.42 \mathrm{~Hz}$ of the spectral knee, which covers the $[0.30-0.75] \mathrm{Hz}$ range, while the vertical dashed-dotted line indicates the proton cyclotron frequency $f_{c p}=1.38 \mathrm{~Hz}$. The PSDs for the field components indicate that the magnetic fluctuations are strongly anisotropic: the dominant contribution is $\mathcal{S}_{y}$ (dashed line, magnetic fluctuations perpendicular to both $\mathbf{B}_{0}$ and $\mathbf{V}_{0}$ ) except in the spectral knee where the spectra $\mathcal{S}_{y}$ and $\mathcal{S}_{x}$ (solid line) are almost identical. The compressive fluctuations, see the spectrum $\mathcal{S}_{z}$ (dashed-dotted line), remain small in the whole frequency range. Thus the fluctuations in the spectral knee are essentially transverse to the mean magnetic field and isotropic in this plane.

[15] The universality of the spectral shapes described here (two power laws and the knee) has to be firmly established through a detailed statistical study. That is the purpose of a future work. However, the agreement with the results of other authors [Czaykowska et al., 2001] indicates that it is likely to be a general property of magnetosheath turbulence, in a broad range of parameters. In the following, we shall restrict ourselves to an analysis of the fluctuations in the spectral knee.

\section{Magnetic Fluctuations in the Spectral Knee Frequency Range}

\subsection{One Satellite Analysis}

\subsubsection{Time Localization}

[16] Let us now use the time resolution of the wavelet transform: we can see from the scalogram (Figure 2b) that the energy is nonuniformly distributed in time and scales. On the scale of the spectral knee $m_{0}=1 / f_{0}=2.37 \mathrm{~s}$ (the horizontal dotted line) one observes a set of energetic peaks localized in time. To select the most energetic events, we consider a section of the scalogram at this scale, $\mathcal{W}^{2}\left(m_{0}, t\right)$, which is shown in Figure 2c.

[17] Figure $2 \mathrm{~d}$ shows a histogram of the wavelet amplitudes $\mathcal{W}\left(m_{0}, t\right)=\sqrt{\mathcal{W}^{2}\left(m_{0}, t\right)}$, still for $m_{0}=2.37 \mathrm{~s}$. This histogram may be compared to the distribution law for the amplitudes of a Gaussian vectorial field (dashed line) with the same standard deviation

$$
\mathcal{P}(\mathcal{W})=\sqrt{\frac{2}{\pi}} \frac{\mathcal{W}^{2}}{\sigma^{3}} \mathrm{e}^{-\mathcal{W}^{2} / 2 \sigma^{2}},
$$

where $\sigma^{2}$ is the variance of $\mathcal{W}\left(m_{0}, t\right)$. The deviation of the actual histogram from the distribution $\mathcal{P}(\mathcal{W})$ is characteristic of some intermittency or inhomogeneity of the turbulence [Frisch, 1995] on the scale $m_{0}$. Events that participate in the energetic non-Gaussian part of the distribution are selected using the threshold value $\mathcal{W}\left(m_{0}\right)=39$ (see the vertical line in Figure 2d). The corresponding threshold energy is shown by a horizontal solid line in
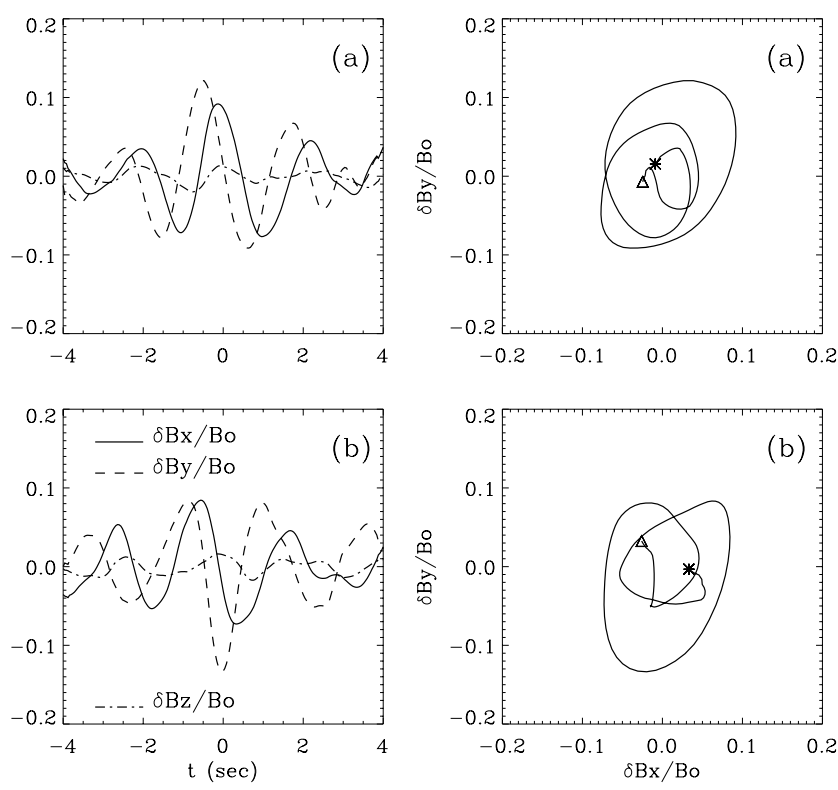

Figure 3. Magnetic waveforms (left) and corresponding hodograms in the plane perpendicular to $\mathbf{B}_{0}$ (right) for the energetic events (a) 3 and (b) 6 of Figure 2c. Asterisks indicate where the hodograms start, and triangles, where they end.

Figure 2c. There are eight events that exceed this threshold energy and we attribute a label to each of them. Later in the text we denote them as "energetic events."

[18] Most of these events (7 out of 8) lie between the scales 1.3 and $3.3 \mathrm{~s}$ in the scalogram of Figure $2 \mathrm{~b}$ but the peak number 8 is significantly larger and covers the scales up to $6 \mathrm{~s}$. It appears that this last event has a different nature than the others and it will not be considered in our analysis. The duration of the events, i.e., the crossing time by the satellite, is in average $8 \mathrm{~s}$, and varies from case to case.

[19] Now, let us look at the structure of the magnetic field in the vicinity of each selected event. Figure 3 (left) shows the time evolution during $8 \mathrm{~s}$ of the three components of the magnetic field fluctuations around peaks 3 (Figure 3a) and 6 (Figure $3 b$ ). The fluctuation of $j$ th component of the magnetic field is defined here by

$$
\delta B_{j}(t)=B_{j}(t)-\overline{B_{j}}(t), \quad j=x, y, z,
$$

where

$$
\overline{B_{j T}}(t)=\frac{1}{N_{T}} \sum_{i=0}^{N_{T}-1} B_{j}\left(t_{i}-t\right)
$$

is a signal smoothed over a time period $T$ (here taken to be $\left.m_{0}\right) . N_{T}=T / \delta t$ is the number of data points within the period $T$. This definition is used to avoid slow trends on scales larger than those of the events we are analyzing.

[20] One can see that the magnetic fluctuations presented on the left side of Figure 3 have regular, well-defined waveforms. The dominant fluctuations occur in the plane perpendicular to $\mathbf{B}_{0}, \delta B_{x} \sim \delta B_{y} \sim 0.1 B_{0}$; the compressible fluctuations are significantly smaller, $\delta B_{z} \sim 0.01 B_{0}$. These 
fluctuations are thus mainly noncompressive. This is in agreement with the results of Figure $2 \mathrm{a}$ and it is confirmed by a minimum variance analysis [Sonnerup and Scheible, 1998] for a $8 \mathrm{~s}$ time period around each selected energetic peak. In all cases, the minimum variance direction $\mathbf{e}_{\min }$ is nearly parallel to the mean magnetic field: the angle between $\mathbf{e}_{\min }$ and $\mathbf{B}_{0}$ lies within the limits $[5-10]^{\circ} \pm 5^{\circ}$.

[21] The two examples presented in Figure 3 (left) look very similar, but there is one important difference, visible in the reference frame based on $\mathbf{B}_{0}$ and $\mathbf{V}_{0}$ : in case a the component along the plasma bulk velocity in the plane perpendicular to $\mathbf{B}_{0}, \delta B_{x}$, is symmetric with respect to the central time of the peak $(t=0)$ and $\delta B_{y}$ is asymmetric; in case $b$ the opposite symmetry is observed. We will see in section 4 the meaning of these different symmetries. For the seven chosen peaks there are six cases similar to case $\mathrm{b}$ and only one with a symmetric $\delta B_{x}$ waveform shown in Figure $3 a$.

[22] The right side of Figure 3 shows the corresponding hodograms $\left(\delta B_{x} / B_{0}, \delta B_{y} / B_{0}\right)$ of the magnetic fluctuations, with asterisks corresponding to the beginning of the hodograms and triangles to their end. In the two cases the polarization is lefthanded with respect to $\mathbf{B}_{0}$ except for a short period of polarization reversal at the end.

[23] These observations on one spacecraft first suggest that the energetic peaks of Figure $2 \mathrm{c}$ are time localized wave packets of Alfvénic fluctuations propagating along $\mathbf{B}_{0}$.

\subsubsection{Alfvénicity}

[24] One expects therefore that the relation between the velocity, and magnetic field fluctuations characteristic of Alfvénic wave modes

$$
\delta V_{\perp} / V_{A}= \pm \delta B_{\perp} / B_{0}
$$

is verified. As said in section 2, the available velocity measurements by CIS experiment [Rème et al., 2001] have a time resolution of $12 \mathrm{~s}$. This is longer than the typical duration of an energetic event and therefore it would seem impossible to check the Alfvénicity of magnetic fluctuations with the CIS data.

[25] However, in Appendix A we describe a method that allows to obtain the velocity fluctuations in the plane perpendicular to $\mathbf{B}_{0}$ with the same time resolution of $0.04 \mathrm{~s}$ as the magnetic and electric fields measurements. The main assumptions of the method are: (1) The Ohm's law $\mathbf{E}=-\mathbf{V} \times \mathbf{B}(\mathbf{E}, \mathbf{B}$ and $\mathbf{V}$ being the electric and magnetic fields and plasma bulk velocity, respectively) is verified over the different timescales involved, from $T \gg$ $1 / f_{c p}$ to $T \sim 1 / f_{c p}$; the Hall term, the electron pressure gradient and the electron inertia remain small. (2) The fields and velocity fluctuations are small with respect to $B_{0}$ and $V_{A}$, respectively. When these assumptions are verified, the two components of the velocity fluctuations in the plane perpendicular to $\mathbf{B}_{0}$ are

$$
\begin{gathered}
\delta V_{x}=\left(\delta E_{y}+V_{0 z} \delta B_{x}-V_{0 x} \delta B_{z}\right) / B_{0} \\
\delta V_{y}=\left(-\delta E_{x}+V_{0 z} \delta B_{y}-V_{0 y} \delta B_{z}\right) / B_{0},
\end{gathered}
$$

where $V_{0 x}, V_{0 y}$, and $V_{0 z}$ are the components of $\mathbf{V}_{0} ; \delta E_{x}$ and $\delta E_{y}$ are the perpendicular components of electric field fluctuations. As explained in section 2, the EFW experiment
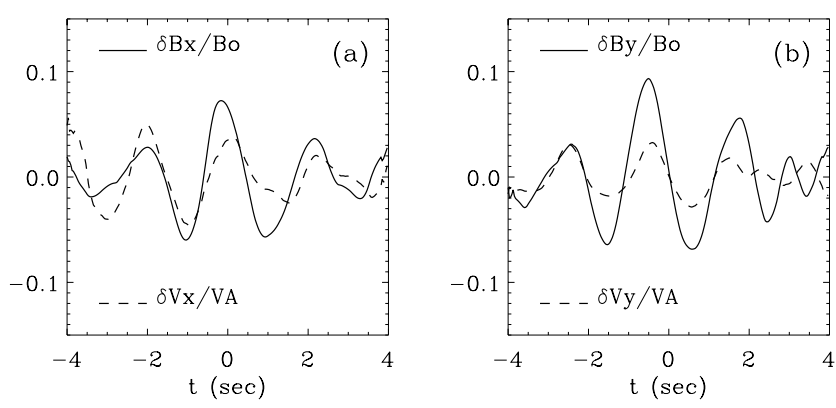

Figure 4. Comparison of the magnetic (solid lines) and velocity (dashed lines) fluctuations in the plane perpendicular to the mean magnetic field for the energetic event number 3: (a) $\delta B_{x} / B_{0}$ and $\delta V_{x} / V_{A}$; (b) $\delta B_{y} / B_{0}$ and $\delta V_{y} / V_{A}$; the velocity fluctuations are determined using the electric and magnetic field data, see expressions (9) and (10).

measures only two electric field components in the GSE frame and the third electric field component is determined from $\mathbf{E} \cdot \mathbf{B}=0$ so that we can compute the electric field in the reference frame based on $\mathbf{B}_{0}$ and $\mathbf{V}_{0}$.

[26] Figure 4 shows the normalized fluctuations of the velocity obtained in this way (dashed line) and of the magnetic field (solid line) in the plane perpendicular to the mean field for the energetic event number 3. Figure $4 \mathrm{a}$ represents $\delta B_{x} / B_{0}$ and $\delta V_{x} / V_{A}$, and Figure $4 \mathrm{~b}$ shows $\delta B_{y} / B_{0}$ and $\delta V_{y} / V_{A}$. In both panels we observe a clear correlation between the magnetic field and velocity fluctuations: typically, the correlation coefficients for the $x$ and $y$ components are $C_{x} \sim C_{y} \sim 0.7-0.8$ for all the energetic events studied here. A linear regression between the magnetic field and velocity fluctuations for the event 3 shown in Figure 4 gives different relations for the two components:

$$
\begin{aligned}
& \delta V_{x} / V_{A}=\xi_{x} \delta B_{x} / B_{0}, \text { with } \xi_{x}=0.5 \\
& \delta V_{y} / V_{A}=\xi_{y} \delta B_{y} / B_{0}, \text { with } \xi_{y}=0.3
\end{aligned}
$$

This difference may be related to measurement problems of the electric field in the direction $X_{\text {gse }}$, due the periodic crossing of the spacecraft shadow by the antenna. Indeed, for the case studied here, $X_{\text {gse }}$ is close to the $x$ axis of the reference frame based on $\mathbf{B}_{0}$ and $\mathbf{V}_{0}$ which we use. This will then affect $\delta V_{y}$ (see expression (10)).

[27] If we now assume that the $x$ component of the velocity is essentially unaffected by this measurement bias, two conclusions follow. The first one is that $\xi_{x}$ is not equal to 1 , the value expected for an Alfvén wave (see equation (8)). The second is that $\xi_{x}$ varies in the range [0.4-0.8] from one event to another. Thus the relation (8) between magnetic and velocity fluctuations must be replaced by a more general one

$$
\delta V_{\perp} / V_{A}=\xi \delta B_{\perp} / B_{0},
$$

with a proportionality coefficient $\xi$ dependant, probably, on the local properties of the plasma and/or of the fluctuation itself.

\subsection{Multisatellite Analysis}

[28] The one satellite study described above suggests that the observed coherent magnetic fluctuations can be inter- 


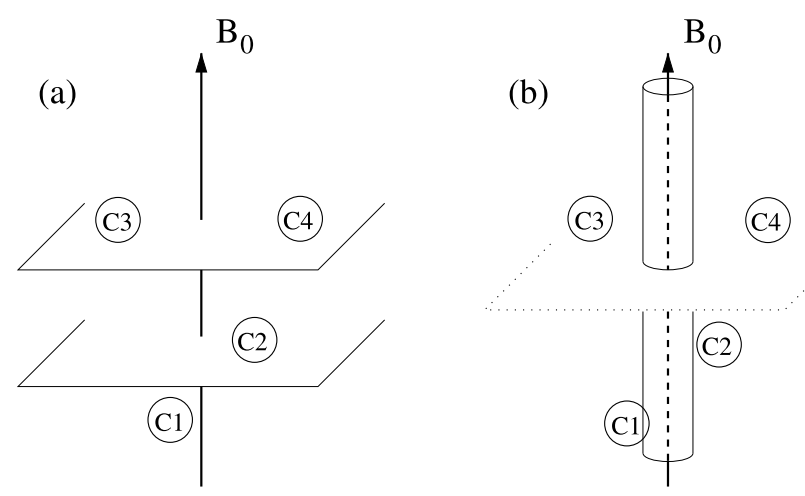

Figure 5. Scheme: (a) a planar wave packet propagating along $\mathbf{B}_{0}$ through the 4 Cluster satellites; (b) an infinite current tube aligned with $\mathbf{B}_{0}$ and localized in the transverse plane.

preted as time localized Alfvénic wave packets propagating along the mean magnetic field, i.e., with the dominant spatial gradients in the direction along $\mathbf{B}_{0}$ (i.e., $k_{\|} \gg k_{\perp}$ ). An alternative interpretation was proposed by Rezeau et al. [1993] and Alexandrova et al. [2004]: each "energetic" event was associated with a current tube with an axis parallel to $\mathbf{B}_{0}$, i.e., with the dominant spatial gradients in the directions perpendicular to $\mathbf{B}_{0}$ (i.e., $k_{\|} \ll k_{\perp}$ ). We shall now see that using the four Cluster spacecraft allows to remove this ambiguity.

3.2.1. Space Localization of the Magnetic Fluctuations

[29] The configuration of the four Cluster satellites with respect to the mean magnetic field on 31 March 2001 at 1700:00 UT is represented schematically in Figure 5. Figure 5a shows planes of constant phase of a wave packet propagating along $\mathbf{B}_{0}$ and passing the four satellites. Figure $5 \mathrm{~b}$ illustrates the other alternative: a cylindrical structure with an axis parallel to $\mathbf{B}_{0}$ and localized in the perpendicular plane.

[30] In the case of the plane wave packet (Figure 5a) the satellites $\mathrm{C} 3$ and $\mathrm{C} 4$ lie roughly in the same wave plane; they observe simultaneously the same signal and, after some delays, the two other satellites observe also this signal.

[31] In the case of a cylindrical structure (Figure 5b), the satellites $\mathrm{C} 3$ and $\mathrm{C} 4$, will eventually observe this structure but with a finite time delay which depends on the satellite separation and the relative velocity of the structure through the satellites. Furthermore, if the structure is roughly uniform along its axis, the satellite $\mathrm{C} 1$ will observe the same signal as C3 and/or C4 with some time delay. (Satellite C2 is located rather far from the others along the $y$ axis, even if it is not apparent in Figure 5, and the probability to observe the same signal on $\mathrm{C} 2$ is small.)

[32] To chose between the two models of Figure 5, we first have to identify the same events on the satellites $\mathrm{C} 1$, $\mathrm{C} 3$, and $\mathrm{C} 4$. The complexity of the energetic events waveforms and the presence of a number of similar fluctuations on all the Cluster satellites complicate the problem of event identification on the different satellites. Some criterion is needed to separate between the observation of the same event by the satellites or the observation of similar but different events. In the first case, i.e., the same event is observed by the satellites $\mathrm{C} i, \mathrm{C} j$, and $\mathrm{C} k$, the time delays $\Delta t_{i j}=t_{j}-t_{i}$, between these satellites should satisfy a compatibility relation

$$
\Delta t_{i j}=\Delta t_{i k}+\Delta t_{k j}, \quad \text { with } \Delta t_{i j}=-\Delta t_{j i} .
$$

If this relation between the time delays is not satisfied, the different satellites observe most probably similar but distinct events. This test is only possible if at least three satellites data are available.

[33] To determine the time delays, we compute the crosscorrelation function

$$
\mathcal{R}_{i j}(\tau)=\frac{\left\langle\delta \mathbf{B}_{i}(t) \cdot \delta \mathbf{B}_{j}(t+\tau)\right\rangle}{\sqrt{\left\langle\delta B_{i}^{2}\right\rangle\left\langle\delta B_{j}^{2}\right\rangle}}
$$

as a function of the time delay $\tau$; the notation $\langle\ldots\rangle$ designs a mean value over a given time interval $T_{t o t}$. The time delay between the observations of the same energetic event on the satellites $\mathrm{C} i$ and $\mathrm{C} j, \Delta t_{i j}$, is then given by the maximum of $\mathcal{R}_{i j}$. This definition involves two distinct time averages: the first one, over the time interval $T$, is used to define the smoothed magnetic field, $\overline{\mathbf{B}}_{T}$, and the corresponding magnetic fluctuations (equations (6), (7)) while the second one, over the time interval $T_{\text {tot }}$, is used to calculate the mean values in equation (13). These two parameters must be adjusted from event to event. Indeed, $T_{\text {tot }}$ has to be chosen so that it is large enough for the different satellites to observe a complete event but small enough to take into account only one event. Similarly, $T$ must be larger that the duration of a typical energetic event but not too large so that the smoothing operation (equation (6)) separates correctly the energetic events from the slower background evolution. Once this choice has been done, the definition (13) is very convenient as it takes into account all the components of magnetic fluctuations and, at the same time, does not depend on the reference frame.

[34] Table 1 gives the values $T$ and $T_{\text {tot }}$ which have been used to analyze each event. We have been able to determine the time delays between the satellites $\mathrm{C} 1, \mathrm{C} 3, \mathrm{C} 4$ for five events out of seven; however, for the peaks 4 and 7 , an unambiguous determination of $\Delta t_{i j}(i, j=1,3,4)$ does not seem feasible by adjusting $T_{\text {tot }}$ and $T$. For the events $1-3,5$, and 6 , the time delays for the satellites $\mathrm{C} 1, \mathrm{C} 3$, and $\mathrm{C} 4$ satisfy the coherency condition (12) with a small error

Table 1. Central Times $t_{0}$, Total Periods Around the Central Times $T_{\text {tot }}$, Smoothing Times $T$, Maximal Correlations Between the Signals Measured by Satellite Pairs C1-C3, C1-C4, C3-C4, and Corresponding Time Lags for the Seven Energetic Events ${ }^{\mathrm{a}}$

\begin{tabular}{ccccccccccc}
\hline Event & $t_{0}, \mathrm{UT}$ & $T_{\text {tot }}$ & $T$ & $\mathcal{R}_{13}$ & $\mathcal{R}_{14}$ & $\mathcal{R}_{34}$ & $\Delta t_{13}$ & $\Delta t_{14}$ & $\Delta t_{34}$ & $\delta \Delta t$ \\
\hline 1 & $1702: 36$ & 12 & 2.25 & 0.9 & 0.7 & 0.7 & -1.52 & 0.63 & 2.11 & 0.04 \\
2 & $1702: 50$ & 11 & 3.00 & 0.8 & 0.6 & 0.7 & -1.48 & 0.78 & 2.27 & 0.00 \\
3 & $1703: 11$ & 12 & 4.50 & 0.8 & 0.6 & 0.6 & -1.52 & 0.78 & 2.23 & 0.08 \\
4 & $1704: 13$ & & & & & & & & & \\
5 & $1704: 40$ & 8 & 2.25 & 0.8 & 0.7 & 0.7 & -1.59 & 1.00 & 2.50 & 0.09 \\
6 & $1705: 31$ & 8 & 2.25 & 0.9 & 0.8 & 0.8 & -1.33 & 1.21 & 2.50 & 0.04 \\
7 & $1705: 38$ & & & & & & & & & \\
\hline
\end{tabular}

${ }^{\mathrm{a}}$ All the time periods are in seconds. 
Table 2. Time Lags for the Three Events Observed by All Cluster Satellites $^{\mathrm{a}}$

\begin{tabular}{cccccccc}
\hline Event & $t_{0}, \mathrm{UT}$ & $\Delta t_{12}$ & $\Delta t_{13}$ & $\Delta t_{14}$ & $\Delta t_{23}$ & $\Delta t_{24}$ & $\Delta t_{34}$ \\
\hline 2 & $1702: 50$ & 1.29 & -1.48 & 0.78 & -2.77 & -0.55 & 2.27 \\
3 & $1703: 11$ & 1.68 & -1.52 & 0.78 & -3.16 & -0.90 & 2.23 \\
6 & $1705: 30$ & -0.47 & -1.33 & 1.21 & -0.82 & 1.68 & 2.50 \\
\hline
\end{tabular}

${ }^{\mathrm{a}}$ All the time delays are measured in seconds.

$\delta \Delta t=\left\|\Delta t_{13}-\left(\Delta t_{14}+\Delta t_{43}\right)\right\|$; see the last column of Table 1. In these cases, the same magnetic structures are most probably observed by the satellites $\mathrm{C} 1, \mathrm{C} 3$, and $\mathrm{C} 4$ and we can study their 3-D geometry.

[35] As one can see from Table 1, the delay $\Delta t_{34}$ varies between $2.1 \mathrm{~s}$ and $2.5 \mathrm{~s}$. As discussed above, a plane wave packet travelling strictly parallel to $\mathbf{B}_{0}$ should be observed simultaneously on $\mathrm{C} 3$ and $\mathrm{C} 4$, which have a separation vector approximately perpendicular to $\mathbf{B}_{0}$. Even if the wave vector makes an angle of $\sim 10^{\circ}$ with $\mathbf{B}_{0}$ (see section 3.1), the satellites $\mathrm{C} 3$ and $\mathrm{C} 4$ should observe the same signal with a time delay of order $0.3 \mathrm{~s}$, an order of magnitude smaller that the observed $\Delta t_{34}$. Therefore the magnetic structure of the energetic peaks $1-3,5$, and 6 is not that of a plane wave packet but is localized in the plane perpendicular to $\mathbf{B}_{0}$.

[36] We see in Table 1 that the correlation $\mathcal{R}_{13}$ between the signals measured on $\mathrm{C} 1$ and $\mathrm{C} 3$ is larger than $\mathcal{R}_{14}$ and $\mathcal{R}_{34}$. As the separation vector between $\mathrm{C} 1$ and $\mathrm{C} 3$ is nearly along the mean magnetic field, the coherent magnetic structures are roughly uniform along $\mathbf{B}_{0}$, at least on scales of the order of the Cluster separation, $600 \mathrm{~km}$.

[37] Thus the analysis of the time delays between the Cluster spacecraft indicates that the energetic peaks of Figure 2c correspond to magnetic coherent structures aligned with $\mathbf{B}_{0}$ and localized in the plane perpendicular to $\mathbf{B}_{0}$ with a cross section smaller than the distance between $\mathrm{C} 3$ and $\mathrm{C} 4$. In the following analysis we assume that the cross section is nearly circular. It could be elliptical as well, but with only four satellites not much more could be deduced.

\subsubsection{Propagation Speed}

[38] With the four Cluster satellites it is possible to determine the velocity $\mathcal{V}$ and the direction of propagation n of a locally planar structure moving with a constant speed in the satellite frame [Schwartz, 1998]

$$
\mathbf{D}_{1 i} \cdot \frac{\mathbf{n}}{\mathcal{V}}=\Delta t_{1 i}, \quad i=2,3,4
$$

Here $\mathbf{D}_{1 i}=\mathbf{D}_{i}-\mathbf{D}_{1}$ is a separation vector between the satellites $\mathrm{C} 1$ and $\mathrm{C} i, \Delta t_{1 i}$ is a temporal delay between measurements on these two satellites. This method, based on time and space separations, is called the timing method. Actually, the timing method keeps its validity (see Appendix B) for cylindrical structures when the following conditions are satisfied: (1) four satellites observe similar signals; (2) the maxima of the six correlation functions $\mathcal{R}_{i j}$ (with $i=1,3$ and $j=2,4$ ) and the corresponding time delays are well defined; (3) the relation (12) is verified for all triplets of satellites.

[39] The timing method can therefore be applied to the coherent structures corresponding to the energetic peaks.
However, only three $(2,3$, and 6$)$ among the five localized structures of Table 1, satisfy the conditions of applicability of the timing method. For these three events Table 2 presents the time delays between the six satellite pairs. One can see indeed that the relation (12) is verified for all triplets of satellites, indicating that these events are observed by the four satellites. The fact that the other events are not observed by the four satellites is consistent with their space localization.

[40] As an example, Figure 6 shows the waveforms for the event number 3 . Here the magnetic fluctuations measured on $\mathrm{C} 2, \mathrm{C} 3$, and $\mathrm{C} 4$ are shifted with respect to the ones observed on $\mathrm{C} 1$ by the corresponding time delays $\Delta t_{1 i}, i=$ $2-4$, and one can see directly that the four satellites observe the same event.

[41] The velocities $\mathcal{V}$ obtained by the timing method (and the corresponding error $\delta \mathcal{V}$ ) for the structures 2,3 , and 6 are given in Table 3. To obtain $\delta \mathcal{V}$, we have taken into account the fact that the satellite separations are determined with an error of 1\% [Credland et al., 1997] and estimated the error on the time delays using the largest deviations about the coherency condition (12). The last line of the table gives the plasma bulk velocity, which is known with a $10 \%$ precision [Rème et al., 2001]. All velocities in this table are projected

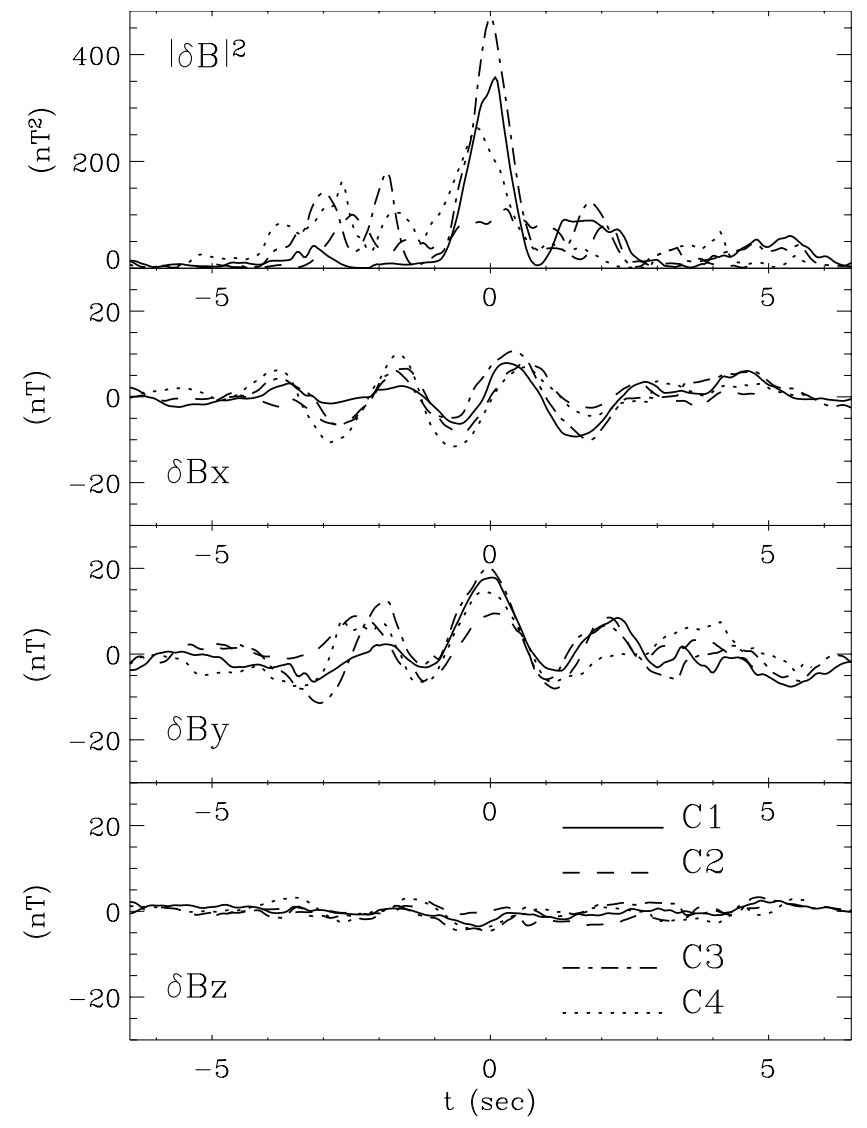

Figure 6. Superposition of magnetic field fluctuations observed around 1703:11 UT by all the Cluster satellites. In the four panels the energy and the three components of the magnetic fluctuations are shown for a $13 \mathrm{~s}$ time period, different line styles indicate different satellites, as shown in the bottom panel. 
Table 3. Results of the Timing Method for the Three Events Observed by All the Cluster Satellites ${ }^{\mathrm{a}}$

\begin{tabular}{cccc}
\hline Event & $t_{0}, \mathrm{UT}$ & $\mathbf{n} \mathcal{V}, \mathrm{km} / \mathrm{s}$ & $(\mathcal{V} \pm \delta \mathcal{V}), \mathrm{km} / \mathrm{s}$ \\
\hline 2 & $1702: 50$ & $(200,-20,-20)$ & $(200 \pm 15)$ \\
3 & $1703: 11$ & $(180,-50,-20)$ & $(190 \pm 20)$ \\
6 & $1705: 30$ & $(190,90,10)$ & $(210 \pm 20)$ \\
& plasma & $(150,0,-100)$ & $(180 \pm 20)$ \\
\hline
\end{tabular}

${ }^{\mathrm{a}}$ The velocities are projected in the reference frame based on $\mathbf{B}_{0}$ and $\mathbf{V}_{0}$. The plasma bulk velocity is given in the last line.

to the reference frame based on $\mathbf{B}_{0}$ and $\mathbf{V}_{0}$; it may be seen that the dominant components of $\mathbf{n} \mathcal{V}$ are perpendicular to $\mathbf{B}_{0}$ while the parallel component, $\mathcal{V}_{z}=(\mathbf{n} \mathcal{V})_{z} \simeq 0$ within the error limits.

[42] In the plane perpendicular to $\mathbf{B}_{0}$ the structures move with respect to the plasma with speeds $\widehat{\mathcal{V}}_{2 \perp}=55 \pm 35 \mathrm{~km} / \mathrm{s}$, $\widehat{\mathcal{V}}_{3 \perp}=60 \pm 40 \mathrm{~km} / \mathrm{s}$, and $\widehat{\mathcal{V}}_{6 \perp}=100 \pm 40 \mathrm{~km} / \mathrm{s}$. Thus structures 2 and 3 are quasi-static and structure 6 moves in the plasma frame with a speed smaller than the local Alfvén velocity, $V_{A} \sim 360 \mathrm{~km} / \mathrm{s}$.

\subsubsection{Characteristic Sizes of the Coherent Structures}

[43] Since the velocities which we have just determined are nearly perpendicular to $\mathbf{B}_{0}$, we can determine the transverse dimensions of the coherent structures. The parallel dimension must be larger than the maximal parallel intrasatellites separation, which is of the order of $600 \mathrm{~km}$.

[44] As seen on the scalogram of Figure 2c, the typical timescale of the localized magnetic fluctuations is $m_{0}=$ $2.37 \mathrm{~s}$ corresponding to a spatial scale $d_{0}=m_{0} \overline{\mathcal{V}}=480 \mathrm{~km}$, with $\overline{\mathcal{V}} \simeq 200 \mathrm{~km} / \mathrm{s}$, the mean speed of the structures with respect to the satellites. This corresponds to $d_{0} \sim 12$ ion inertial length $c / \omega_{p i}$ or $d_{0} \sim 23$ ion thermal gyroradius $\rho_{i}\left(\rho_{i}=\right.$ $V_{T i} / \Omega_{c i}$, where $V_{T i}=\sqrt{k_{B} T_{i} / m_{i}}=185 \mathrm{~km} / \mathrm{s}$ is the ion thermal velocity and $\Omega_{c i}=2 \pi f_{c i}$ ).

[45] In the magnetosheath, where $\beta_{i} \propto \rho_{i}^{2} /\left(c / \omega_{p i}\right)^{2}$ is in general of the order of one, it is difficult to distinguish between the ion inertial scale and the Larmor radius, although their variations on long times may be different. This is illustrated in the upper panel of Figure 7. The two characteristic lengths are transformed into timescales by dividing by $V_{b \perp}$, the plasma bulk velocity in the plane perpendicular to $\mathbf{B}_{0}$ and their time evolution is superimposed on the scalogram of the Morlet wavelet of the component $B_{X g s e}$ of the magnetic field for a period of $82 \mathrm{~min}$ starting at 1642:00 UT on 31 March 2001. This period is much longer than the 4 min time period studied in this paper (shown by the two vertical dotted lines). The "Larmor scale" $\rho_{i} / V_{b \perp}$ is the yellow line; the "ion inertial scale" $\left(c / \omega_{p i}\right) / V_{b \perp}$ (multiplied by a factor of 4 to separate the two lines) is the red line. The lower panel of the figure displays the evolution of the approximate distance $d$ from Cluster to the shock front, obtained by using the shock position determined by Maksimovic et al. [2003]. The shock crossings correspond to $d=0$. In the solar wind $d<0$ and in the magnetosheath $d>0$.

[46] For each magnetosheath crossing, the scalogram exhibit intense emission peaks at scales $m_{0}$ which depend slowly on time (the corresponding periods being $\sim 1-3 \mathrm{~s}$ ). These are the time-localized coherent magnetic fluctuations described above. It may be seen that (1) their scale $m_{0}$ changes with the distance from the shock: it being larger when the Cluster satellites are deeper into the magnetosheath, (2) the time evolution of $m_{0}$ is similar to that of $c / \omega_{p i} V_{b \perp}$ and somewhat different from the evolution of the Larmor scale $\rho_{i} / V_{b \perp}$. This indicates that $m_{0}$ scales with the ion inertial scale rather than with the Larmor radius.

[47] An other important conclusion that can be made from the analysis of the scalogram of Figure 7 is related to the occurrence of the coherent time-localized magnetic fluctuations in the magnetosheath. Indeed, these fluctuations are observed during the whole second and the third magnetosheath crossings. Before the first shock crossing they appear only at $t \simeq 15 \mathrm{~min}$, when Cluster left the cusp region to enter the magnetosheath as discussed in section 2. Thus the field-aligned magnetic structures seem to fill the whole magnetosheath region for this particular day suggesting that these structures are stable in the magnetosheath plasma.

\subsection{Summary of the Observations}

[48] The time localized coherent magnetic fluctuations observed at time scales $\sim 1-3 \mathrm{~s}$, which look like parallel propagating Alfvén wave packets, when observed with only one spacecraft, are indeed field-aligned structures, localized in the plane perpendicular to the mean magnetic field, $k_{\|} \ll$ $k_{\perp}$, with a transverse dimension of the order of $10 \mathrm{c} / \omega_{p i}$. They have an Alfvénic nature and appear to be largely incompressible. Their proper velocity in the plasma frame is perpendicular to $\mathbf{B}_{0}$ and is much smaller than the local Alfvén speed.

[49] The incompressible MHD equations have localized three-dimensional solutions, called "Alfvén vortices" [Petviashvili and Pokhotelov, 1992], which have also an Alfvénic nature (see equation (11)), which are stationary in the plasma if their axis is aligned with the unperturbed magnetic field, $\mathbf{B}_{0}$, or move slowly in the plasma, while keeping their shape, if their axis makes a small angle with

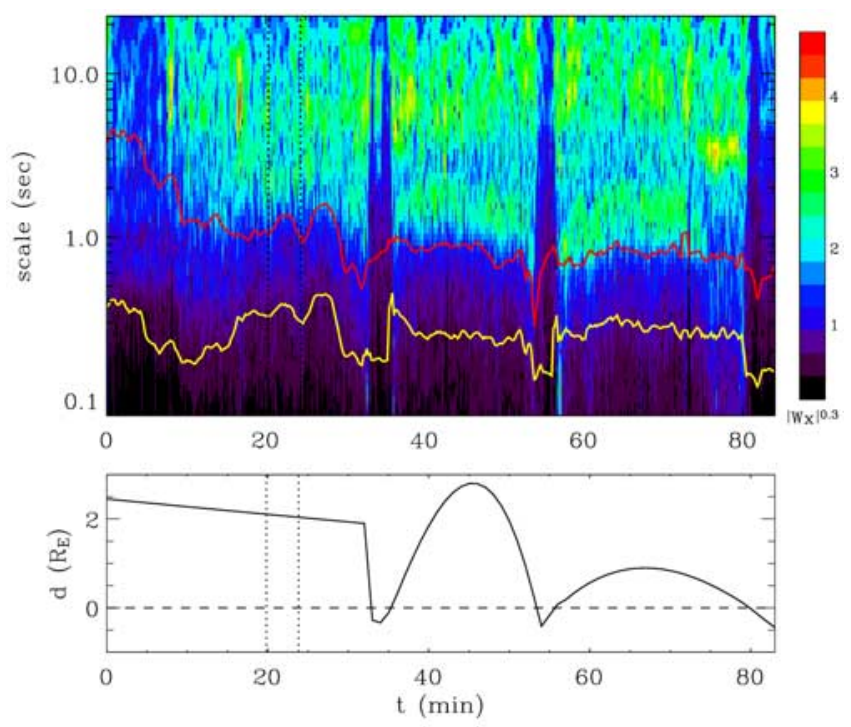

Figure 7. (top) Morlet scalogram of $B_{\text {Xgse }}$ for the period of $82 \mathrm{~min}$, from 1642:00 UT on 31 March 2001. Variations of $c / \omega_{p i}$ (red line) and $\rho_{i}$ (yellow line). (bottom) Distance to the shock from the Cluster satellite $d$ for the same time period; $d$ is determined using the results of Maksimovic et al. [2003]. 
$\mathbf{B}_{0}$. We shall now see that the properties of the localized structures described above compare favorably with what one would expect from Alfvén vortices.

\section{Alfvén Vortex}

[50] First, let us summarize briefly the main theoretical features of the Alfvén vortex model [Petviashvili and Pokhotelov, 1992]. It is based on the ideal, incompressible, MHD equations. The magnetic field and velocity fluctuations are mostly perpendicular to the unperturbed magnetic field $\mathbf{B}_{0}, \delta B_{z} \ll \delta B_{\perp}$ and $\delta V_{z} \ll \delta V_{\perp}$, where, as before, the $z$ direction is parallel to $\mathbf{B}_{0}$; they have a slow time dependence, $\partial_{t} \ll \Omega_{c i}$, and their space variations is faster perpendicular to $\mathbf{B}_{0}$ than parallel to it, $\partial_{z} \ll \nabla_{\perp}$. Their amplitude $\varepsilon \sim \delta B_{\perp} / B_{0}$ is assumed to be small although finite, $0<\varepsilon<1$ and they satisfy the following scaling relations:

$$
\frac{\partial_{z}}{\nabla_{\perp}} \sim \frac{\partial_{t}}{V_{A} \nabla_{\perp}} \sim \frac{\delta B_{z}}{B_{\perp}} \sim \frac{\delta V_{z}}{\delta V_{\perp}} \sim \frac{\delta B_{\perp}}{B_{0}} \sim \frac{\delta V_{\perp}}{V_{A}} \sim \varepsilon
$$

Note that these relations correspond well to the observations (see section 3 and subsections 3.1 and 3.2.1).

[51] The transverse fluctuations can then be described only by two scalar functions, the parallel component of the vector potential $\mathbf{A}$ and by a flux function $\psi$ :

$$
\delta \mathbf{B}_{\perp}=\nabla A_{z} \times \mathbf{z}, \quad \delta \mathbf{V}_{\perp}=\mathbf{z} \times \nabla \psi .
$$

For these two scalar variables the MHD equations reduce to two scalar equations [Kadomtsev and Pogutse, 1974; Strauss, 1976; Petviashvili and Pokhotelov, 1992], the conservation of the momentum along $z$ and the MaxwellFaraday equation in the plane perpendicular to $z$. These equations can be written in dimensionless form, using new variables $t=\Omega_{c i} t, r_{\perp}=r_{\perp} / \rho_{i}, z=z /\left(c / \omega_{p i}\right), \rho=\rho / \rho_{0}, \Phi=$ $\psi /\left(\rho_{i}^{2} \Omega_{c i}\right), A=A_{z} V_{A} /\left(B_{0} \rho_{i}^{2} \Omega_{c i}\right)$

$$
\begin{gathered}
d_{t} \nabla_{\perp}^{2} \Phi=\{A, J\}-\partial_{z} J \\
d_{t} A+\partial_{z} \Phi=0,
\end{gathered}
$$

where $J=\nabla_{\perp}^{2} A$ is the longitudinal current,

$$
d_{t} \equiv \partial_{t}+\delta \mathbf{V}_{\perp} \cdot \nabla_{\perp}
$$

and the notation $\{.,$.$\} corresponds to the Poisson brackets$ (or the Jacobian)

$$
\{a, b\}=\partial_{x} a \partial_{y} b-\partial_{y} a \partial_{x} b=(\nabla a \times \nabla b) \cdot \mathbf{z} .
$$

Note that the dependence on $z$ is kept in the equations (17) and (18) in the terms with $\partial_{z}$.

[52] The Alfvén vortices are solutions which have a finite size in a plane nearly perpendicular to $z$ and propagate with a speed $u$ in this plane while conserving their shape. They depend only on two variables $x$ and $\eta$, with

$$
\eta=y+\alpha z-u t, \quad \alpha=\tan (\vartheta)
$$

$\vartheta$ being the angle between the normal to the plane $(x, \eta)$ and $\mathbf{B}_{0}$. For the condition $\partial_{z} \ll \nabla_{\perp}$ to be satisfied, the angle must be small, $\alpha \sim \partial_{z} / \nabla_{\perp} \sim \varepsilon$. Similarly, the velocity $u$ must be also small in order to satisfy the condition $\partial_{t} \ll \Omega_{c i}$, i.e., $u \sim \partial_{t} / \Omega_{c i} \sim \varepsilon$. Instead of the angle $\vartheta$ (or $\alpha$ ), it is convenient to use the variable $\xi$

$$
\xi=\frac{u}{\alpha}, \quad \text { with } u \sim \alpha \sim \varepsilon
$$

In principle, $\xi$ is arbitrary but of the order of 1 .

[53] In the new variables $(x, \eta)$ the equations (17) and (18) become

$$
\begin{gathered}
\left\{\Phi-u x, \nabla_{\perp}^{2}(\Phi-u x)\right\}=\left\{A-\frac{u}{\xi} x, J\right\} \\
\left\{\Phi-u x, A-\frac{u}{\xi} x\right\}=0
\end{gathered}
$$

with the new Poisson bracket $\{a, b\}=\partial_{x} a \partial_{\eta} b-\partial_{\eta} a \partial_{x} b$. Equation (22) means that $(\Phi-u x)$ and $\left(A-\frac{u}{\xi} x\right)$ are dependent on one another:

$$
A-\frac{u}{\xi} x=f(\Phi-u x)
$$

so that equation (21) leads to an equation for $(\Phi-u x)$

$$
\nabla_{\perp}^{2}(\Phi-u x)=f^{\prime}(\Phi-u x) J+f_{1}(\Phi-u x)
$$

containing two arbitrary functions, $f$ and $f_{1}$. Therefore there is an infinite number of solutions of the system (21) and (22).

[54] Among this infinite set of solutions, the Alfvén vortex satisfies a generalized Alfvén relation (see equation (11))

$$
\Phi=\xi A
$$

while the corresponding current density $J$ is a linear function of $A-\frac{u}{\xi} x$ inside a circle of radius $a$ and vanishes outside

$$
\left\{\begin{array}{l}
J=-k^{2}\left(A-\frac{u}{\xi} x-\hat{c}\right), r<a \\
J=0, r \geq a
\end{array},\right.
$$

where $k$ and $\hat{c}$ are constants.

[55] The arbitrary functions appearing in equation (24) are then specified and the solution that decays at infinity as a power law is

$$
\left\{\begin{array}{l}
A=A_{0}\left(J_{0}(k r)-J_{0}(k a)\right)+\frac{u}{\xi} \frac{x}{r}\left(r-2 \frac{J_{1}(k r)}{J_{0}(k a)}\right), r<a \\
A=a^{2} \frac{u}{\xi} \frac{x}{r^{2}}, r \geq a
\end{array}\right.
$$

Here $A_{0}$ is a constant amplitude, $J_{0}$ and $J_{1}$ are the Bessel functions of zeroth and first order, respectively, $r=$ $\sqrt{x^{2}+\eta^{2}}$ is the radial variable in the plane of the vortex. 

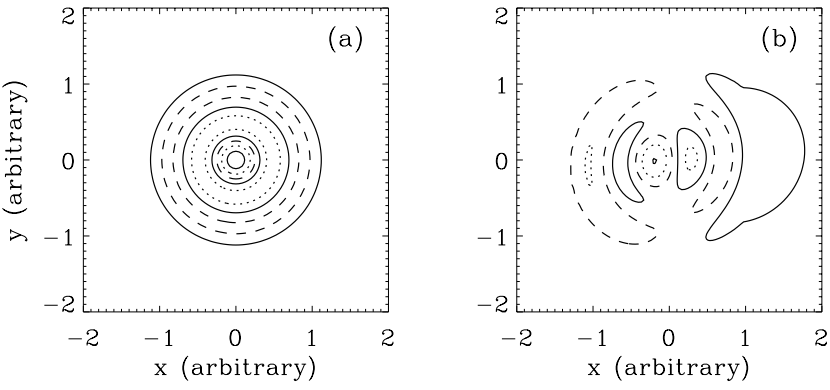

Figure 8. Magnetic field lines of Alfvén vortices in the plane perpendicular to $\mathbf{B}_{0}$ for $z=0$; (a) a field-aligned vortex (monopole), (b) a vortex with an axis inclined with an angle of $4^{\circ}$ and that propagates with $u=0.07 V_{A}$ along the $y$ axis.

[56] The continuity of the solution (27) in $r=a$ requires that the parameter $k$ and the radius $a$ be coupled by the following dispersion relation

$$
J_{1}(k a)=0 .
$$

This relation ensures the continuity of the radial magnetic field in $r=a$ as well as a vanishing divergence of $\delta \mathbf{B}_{\perp}$ everywhere.

[57] Since

$$
\delta \mathbf{B}_{\perp}=\left(\partial_{y} A_{z},-\partial_{x} A_{z}\right)=\left(\partial_{\eta} A,-\partial_{x} A\right)
$$

the components of the magnetic field fluctuation in the plane perpendicular to $\mathbf{B}_{0}$ for $r<a$ are given by

$$
\begin{gathered}
\delta B_{x}=k A_{0} J_{0}^{\prime}(k r) \frac{\eta}{r}+\frac{u}{\xi} \frac{2}{J_{0}(k a)}\left(\frac{J_{1}(k r)}{r}-k J_{1}^{\prime}(k r)\right) \frac{x \eta}{r^{2}} \\
\delta B_{y}=-k A_{0} J_{0}^{\prime}(k r) \frac{x}{r}+\frac{u}{\xi} \frac{2}{J_{0}(k a)}\left(\frac{J_{1}(k r)}{r} \eta^{2}+k J_{1}^{\prime}(k r) x^{2}\right) \frac{1}{r^{2}}-\frac{u}{\xi} .
\end{gathered}
$$

Here, the prime indicates the derivation with respect to the argument. In the outside region the field is

$$
\begin{gathered}
\delta B_{x}=-2 a^{2} \frac{u}{\xi} \frac{x \eta}{r^{4}} \\
\delta B_{y}=2 a^{2} \frac{u}{\xi}\left(\frac{x^{2}}{r^{4}}-\frac{1}{2 r^{2}}\right) .
\end{gathered}
$$

If $u=0$, the field is zero outside of the circle of radius $a$.

[58] The magnetic field lines in the plane $(x, y)$ are represented in Figure 8. Here we have chosen the following parameters: $A_{0}=-0.15, \hat{c}=0, a k=10.17$, that correspond to the third zero of the Bessel function $J_{1}$. Figure $8 \mathrm{a}$ corresponds to the field-aligned vortex. Figure $8 \mathrm{~b}$ corresponds to the vortex that is inclined with an angle of $4^{\circ}$ with respect to $z$ and that moves with velocity $u=0.07$
( $u$ is normalized on $V_{A}$ ) in the direction of a nonzero mean magnetic field projection on the vortex plane (see expression (19)).

[59] As one can see from Figure 8a, the magnetic field lines of the field-aligned vortex have a cylindrical symmetry with respect to the vortex center: it is a monopolar vortex. The inclined vortex is no longer symmetric, see Figure $8 \mathrm{~b}$ and has a bipolar structure.

[60] Figure 9 shows "measured" magnetic field fluctuations by a "simulated" satellite passing by these two vortices along the $x$ direction at a finite distance from their centers $(y \neq 0)$. In Figure 9a, we can see that the component along the satellite trajectory, $\delta B_{x}$, is a symmetric function with respect to the vortex center and $\delta B_{y}$ is an antisymmetric one. The radial magnetic field

$$
\delta B_{r}=\delta B_{x} \frac{x}{r}+\delta B_{\eta} \frac{\eta}{r}=\delta B_{x} \frac{x}{r}+\delta B_{y} \frac{y}{r}
$$

remains zero all along the satellite trajectory. In Figure $9 \mathrm{~b}$ the radial field is not zero, it is symmetric with respect to the center, $\delta B_{x}$ is nearly antisymmetric, and $\delta B_{y}$ is nearly symmetric.

[61] In section 3 we could distinguish two types of coherent structures in the reference frame of $\mathbf{V}_{0}$ and $\mathbf{B}_{0}$, see Figure 3 (left). The structure of Figure $3 a$ (event 3 ) is similar to the fluctuations of the monopolar vortex shown in Figure 9a; and the structure of Figure $3 \mathrm{~b}$ (event 6 ) is similar to the dipole of Figure $9 \mathrm{~b}$. As we have seen in section 3.2.2, the structure 3 can be quasi-stationary in the plasma, and the structure 6 has a finite velocity, but smaller than $V_{A}$. This is qualitatively in agreement with the properties of the two vortex types.

[62] Since the variations of the field have scales much longer parallel to the structure axis than perpendicular to it, the direction of minimum variance as calculated in section 3 should give an estimate of the direction of this axis and of its inclination with respect to the mean magnetic field. In section 3 we have seen that the angle between $\mathbf{e}_{\min }$ and $\mathbf{B}_{0}$ for the different events varies from 5 to 10 degrees. The fact that the angles are small is in agreement with the Alfvén vortex model. However, they are of the same order as the errors on their measurement, and it does not appear possible to proceed further in this direction and to study whether the observed symmetry of magnetic fluctuations depends on the angle or not.
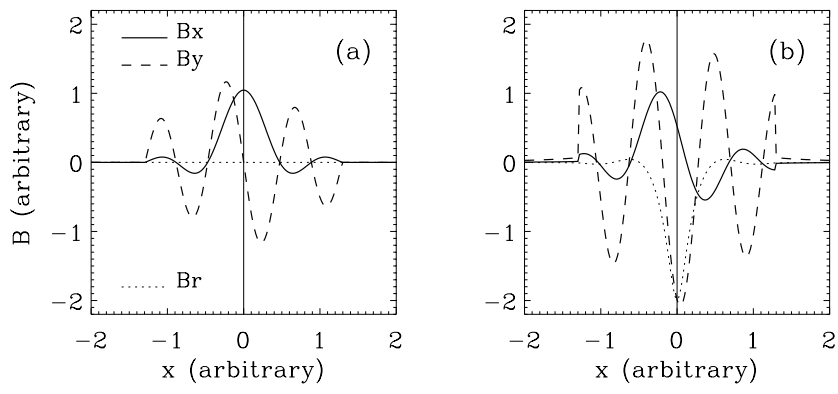

Figure 9. Magnetic field fluctuations measured by a simulated satellite passing by the two vortices presented in Figure 8 along the $x$-axis; (a) monopole and (b) dipole. 

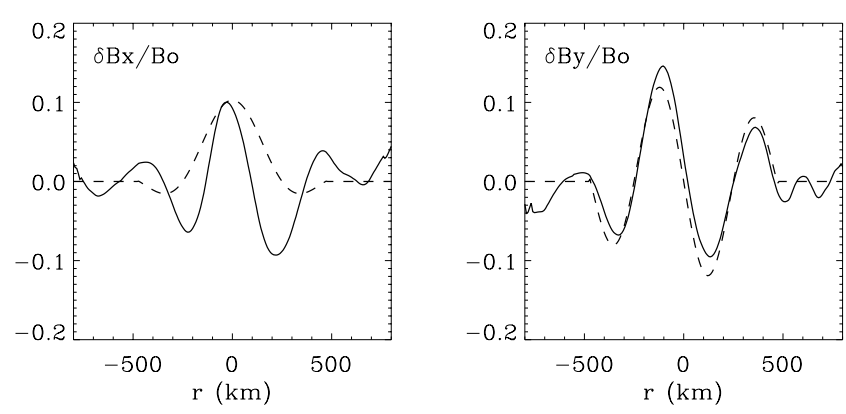

Figure 10. Comparison between the fluctuations of the event 3 (solid lines) and the model of the monopole Alfvén vortex (dashed lines).

[63] One may still compare the magnetic variations of the structures 3 and 6 with those expected from the crossing of an Alfvén vortex. For the structure 3, magnetic fluctuations are fitted with the monopole vortex model; i.e., in the expressions (30)-(33) we take $u=0$

$$
\begin{gathered}
\delta \mathbf{B}_{\perp}=\left(k A_{0} J_{0}^{\prime}(k r) \frac{\eta}{r},-k A_{0} J_{0}^{\prime}(k r) \frac{x}{r}\right), r<a \\
\delta \mathbf{B}_{\perp}=0, r \geq a,
\end{gathered}
$$

corresponding to a force-free or magnetostatic current filament of radius $a$. Varying the value of the relative amplitude $A_{0} k$ of the fluctuations in the vortex center, the parameter $k$ and the minimal distance $h$ (impact parameter) of the satellite trajectory with respect to the center, we obtain the fitting presented in Figure 10. Here the observed fluctuations are shown by the solid lines and the results of the fit, by the dashed ones. The model parameters which fit best the observations are the following: the amplitude $k A_{0}=$ -0.24 , the Bessel function parameter $1 / k \simeq 70 \mathrm{~km}$, which is of order of the protons inertia length $\left(c / \omega_{p i} \simeq 40 \mathrm{~km}\right)$. The impact parameter is $h \simeq 65 \mathrm{~km}$ and the radius of localization $a \simeq 470 \mathrm{~km}$, that corresponds to the second zero of the Bessel function $J_{1}, k a \simeq 7$ (see expression (28)). Time variations of the observed magnetic structures are transformed into the spatial variations of the vortex model by using the structure velocity with respect to the satellite (see section 3.2.2). As we have seen in section 3.2.3, the characteristic scale of the coherent structures is $d_{0}=$ $480 \mathrm{~km}$. The parameter $a$ determined here is of the same order of magnitude.

[64] The waveforms of the structure 6 are fitted with the bipolar vortex model, see Figure 11 . Here the parameters are the following: $k A_{0}=-0.03,1 / k \simeq 70 \mathrm{~km}, h \simeq 100 \mathrm{~km}$, the radius of localization $a \simeq 500 \mathrm{~km}, k a \simeq 7$ and the angle of the vortex inclination is $\vartheta \sim 1^{\circ}$.

[65] One can see from Figures 10 and 11 that the observed waveforms with different symmetries, described in section 3, are well represented by the monopole and the dipole Alfvén vortex models.

\section{Conclusion and Discussion}

[66] In this paper we have demonstrated for the first time the presence of Alfvén vortex filaments in the magneto- sheath turbulence downstream of a quasi-perpendicular bow shock. The corresponding magnetic fluctuations appear in the power spectrum as a large maximum and participate to the intermittency of the magnetosheath turbulence. The polarization of these fluctuations, the minimum variance direction and the magnetic field/velocity relationship correspond to Alfvénic fluctuations. That is why these fluctuations and the spectrum knee were previously interpreted as AIC waves, generated by unstable anisotropic ion distributions [Czaykowska et al., 2001].

[67] The multisatellite analysis performed here shows without any ambiguity some new properties of these Alfvénic fluctuations, not coherent with the plane wave interpretation. Namely, these fluctuations are localized in the plane perpendicular to the minimum variance direction. If the minimum variance direction is parallel to $\mathbf{B}_{0}$, the propagation velocity determined from the four satellites timing method is nearly perpendicular to $\mathbf{B}_{0}$ and its magnitude in the plasma frame is much smaller than the local Alfvén speed. These characteristics indicate that the Alfvénic fluctuations are not plane waves but Alfvén vortex filaments, which are three-dimensional nonlinear structures.

[68] The identification and detailed analysis of the Alfvén vortices reported in this paper was possible thanks to a fortunate configuration of the Cluster spacecraft. Two satellites have a separation vector nearly perpendicular to the unperturbed magnetic field and all the separations are larger but of the order of the Alfvén vortex perpendicular dimension. When the Cluster separations are much larger than the scale of the vortex, the probability to find the same event on the four satellites is very small. If the satellites are very close to each other, the time delays between the observations on the different satellites are so small that it is not possible to determine them correctly, and thus the analysis of the 3-D structure of the fluctuations is impossible.

[69] A generation mechanism of the Alfvén vortex filaments in the magnetosheath is an open question. Several possibilities may be considered. Drift Alfvén vortices [Shukla et al., 1985], which are solutions of generalized Hasegawa-Mima equations, are similar to those described above. They are generated naturally in a plasma with strong gradients, when the drift velocity of the particles $V_{d}=-\nabla p \times \mathbf{B} /$ ne $^{2}$ becomes comparable to their thermal velocity [Petviashvili and Pokhotelov, 1992].
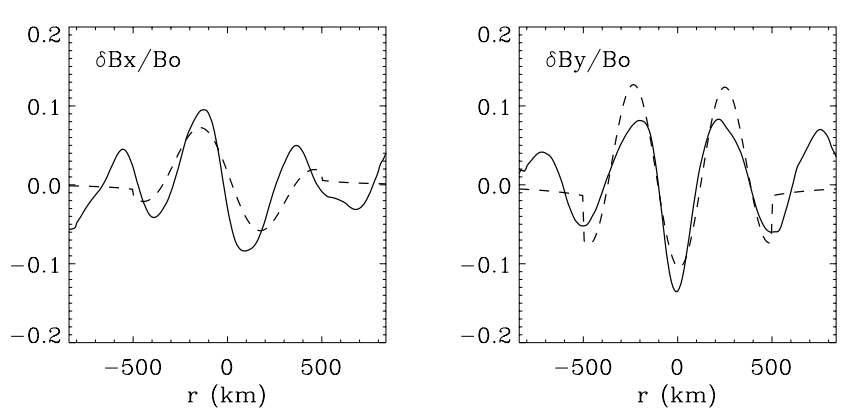

Figure 11. Comparison between the fluctuations of the event 6 (solid lines) and the model of the dipole Alfvén vortex (dashed lines). 
The perpendicular size of the drift Alfvén vortices is of the order of the ion Larmor radius. This kind of vortex structures has been observed in the ionosphere [Chmyrev et al., 1988] by the IC-B-1300 satellite and more recently in the magnetospheric cusp region [Sundkvist et al., 2005] by the Cluster satellites. However, in the magnetosheath plasma the drift velocity of the ions is of the order of $1 \mathrm{~km} / \mathrm{s}$, which is negligible with respect to all the characteristic velocities in the medium, and the characteristic scale of the observed vortices is shown to scale with the ion inertial length rather than with the proton Larmor radius. Therefore it is unlikely that the coherent structures we have described here are drift Alfvén vortices.

[70] A more appropriate possibility is that the vortices are the result of the development of a turbulent cascade. Assuming that the magnetosheath turbulence is weak, since $\delta B / B_{0}<1$, the statistics of the magnetic field, at large scale, should be nearly Gaussian. It has been shown by several authors [see, e.g., Galtier et al., 2002] that the approximation of weak turbulence fails at small scales where some intermittence develops. The nature of the corresponding coherent structures remains an open problem but one may speculate that in the magnetosheath conditions, they take the form of Alfvén vortices. Indeed, in the presence of a finite large-scale magnetic field, the cascade proceeds mainly perpendicularly to the mean magnetic field, i.e., $k_{\perp} \gg k_{\|}$. The limit $k_{\|} \rightarrow 0$, corresponds to a very long correlation length along the mean field as in a field-aligned monopolar Alfvén vortex. The fact that we observe much more inclined vortices with a small angle (bipolar vortices with a finite $k_{\|}$) could indicate that the turbulence avoids the longest correlations.

[71] Numerical simulations of the strong MHD turbulence [Shebalin et al., 1983; Grappin, 1986; Matthaeus et al., 1996; Müller and Grappin, 2005; Dmitruk et al., 2005] and of the Hall MHD turbulence [Ghosh and Goldstein, 1997] show the development of the anisotropic cascade, $k_{\perp} \gg k_{\|}$, in the presence of a mean field. Such a bidimensional turbulence tends to a state where the fluctuations of the velocity and magnetic fields are aligned [Ting et al., 1986] as in the Alfvén vortex.

[72] The bidimensional fluctuations with $k_{\perp} \gg k_{\|}$have already been observed in the solar wind turbulence [Matthaeus et al., 1990]. Can these fluctuations be at the origin of the magnetosheath vortices? The Earth bow shock represents an obstacle for the solar wind fluctuations. A detailed study of the transformation of the 2-D solar wind fluctuations across the shock discontinuity must be done in order to answer this question. However, the bow shock itself can play a crucial role in the vortex formation. For neutral fluids it was shown that the vorticity jump across the shock is very large when the curvature of the shock is important, and it leads to the formation of a vortex sheet downstream of the shock [Kevlahan, 1997].

[73] Another possible generation mechanism of Alfvén vortices in the magnetosheath can be the Alfvén wave filamentation instability [Laveder et al., 2002; Passot and Sulem, 2003]. This is suggested by the observations described by Alexandrova et al. [2004], where monochromatic AIC waves are observed in regions close to where fieldaligned filaments (here shown to be the Alfvén vortices) are found. However, monochromatic waves are not frequently observed during the $82 \mathrm{~min}$ time period of Figure 7 , in opposition to Alfvén vortices. This could be due to the fact that the filamentation process is so fast that we observe only its result and not the initial AIC wave. The importance of filamentation instability in vortex formation must be verified by numerical simulations on the one hand and by a global statistical analysis of magnetosheath crossings on the other hand.

\section{Appendix A: Determination of the Velocity Fluctuation}

[74] Here we propose a method which allows to obtain the first moment of the ion distributions in the plane perpendicular to the background magnetic field $\mathbf{B}_{0}$ with the same time resolution as the field fluctuations. This method is based on the Ohm's law of the ideal magnetohydrodynamics (MHD):

$$
\mathbf{E}=-\mathbf{V} \times \mathbf{B}
$$

We suppose that the measured electric field verifies Ohm's law (A1) on the timescales between $T \gg 1 / f_{c p}$ and the proton cyclotron scale $T \sim 1 / f_{c p}$. To take into account the different scales, we write

$$
\mathbf{B}=\mathbf{B}_{\mathbf{0}}+\delta \mathbf{B} ; \quad \mathbf{V}=\mathbf{V}_{0}+\delta \mathbf{V},
$$

therefore the electric field (A1) is

$$
\begin{aligned}
\mathbf{E} & =-\left[\left(\mathbf{V}_{0}+\delta \mathbf{V}\right) \times\left(\mathbf{B}_{\mathbf{0}}+\delta \mathbf{B}\right)\right] \\
& =-\left[\mathbf{V}_{\mathbf{0}} \times \mathbf{B}_{\mathbf{0}}\right]-\left[\delta \mathbf{V} \times \mathbf{B}_{\mathbf{0}}\right]-\left[\mathbf{V}_{0} \times \delta \mathbf{B}\right]-[\delta \mathbf{V} \times \delta \mathbf{B}]
\end{aligned}
$$

[75] We suppose that the term $[\delta \mathbf{V} \times \delta \mathbf{B}]$ is small with respect to the other terms and we define

$$
\begin{gathered}
\mathbf{E}_{\mathbf{0}}=-\mathbf{V}_{\mathbf{0}} \times \mathbf{B}_{\mathbf{0}} \\
\delta \mathbf{E}=-\left[\delta \mathbf{V} \times \mathbf{B}_{\mathbf{0}}\right]-\left[\mathbf{V}_{\mathbf{0}} \times \delta \mathbf{B}\right] .
\end{gathered}
$$

Now it is convenient to chose a reference frame in which $\mathbf{B}_{0}=\left(0,0, B_{0}\right)$. We will call it "magnetic field frame." In this reference frame (A4) component by component is

$$
\left\{\begin{array}{l}
\delta E_{x}=-\delta V_{y} B_{0}-V_{0 y} \delta B_{z}+V_{0 z} \delta B_{y} \\
\delta E_{y}=\delta V_{x} B_{0}+V_{0 x} \delta B_{z}-V_{0 z} \delta B_{x} \\
\delta E_{z}=-V_{0 x} \delta B_{y}+V_{0 y} \delta B_{x}
\end{array}\right.
$$

The first and second equations of this system give the velocity fluctuations in the plane perpendicular to $\mathbf{B}_{0}$ as functions of the field's fluctuations, the background magnetic field, and plasma bulk velocity:

$$
\left\{\begin{array}{l}
\delta V_{x}=\frac{1}{B_{0}}\left(\delta E_{y}+V_{0 z} \delta B_{x}-V_{0 x} \delta B_{z}\right) \\
\delta V_{y}=\frac{1}{B_{0}}\left(-\delta E_{x}+V_{0 z} \delta B_{y}-V_{0 y} \delta B_{z}\right)
\end{array} .\right.
$$



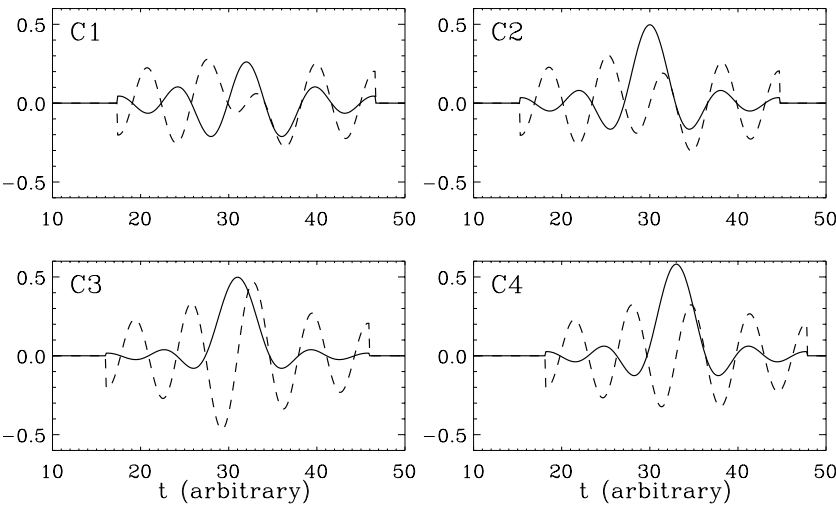

Figure B1. Four synthetic signals measured by the modelled satellites: $\delta B_{x}$ (solid lines) and $\delta B_{y}$ (dotted lines).

The third equation of system (A5) gives an expression for the parallel fluctuation of the electric field that is expected to be small in the ideal MHD description.

\section{Appendix B: Timing Method for the Cylindrical Magnetic Structure}

[76] In this appendix we show using a numerical experiments that the timing method [Schwartz, 1998] can be applied to a cylindrical structure, such as a field-aligned current (or an Alfvén vortex) in order to determine its velocity. We discuss as well the limits of this method.

[77] We consider the model of a field-aligned current (or a monopole vortex) described by equations (34) and (35). The trajectory of the satellite $\mathrm{C} j$ with a constant speed through the current is $\mathbf{r}_{j}=\mathbf{r}_{0 j}+\mathbf{V} t$. We chose $\mathbf{V}=(1,0,0)$ and we suppose that this speed is the same for all the satellites. The synthetic signals on the four satellites are shown in Figure B1.

[78] To apply the timing method to these synthetic signals, we need to know the time and space separations between the satellites. In the in situ observations the satellites separations are well known. It is the determination
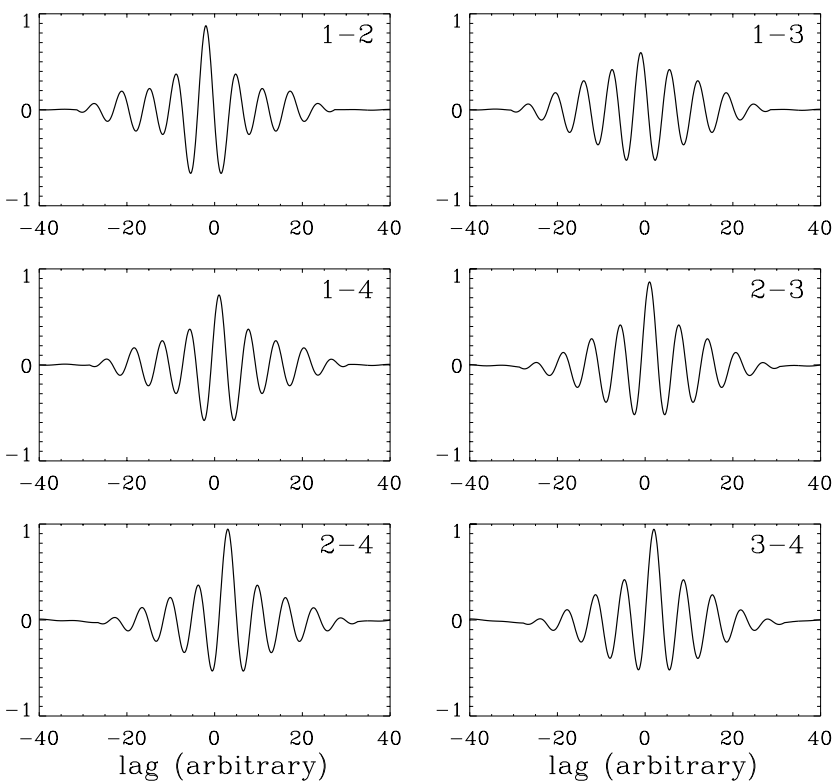

Figure B2. Cross-correlations $\mathcal{R}_{i j}$, with $i=1-4$ and $j=$ $2-4$.
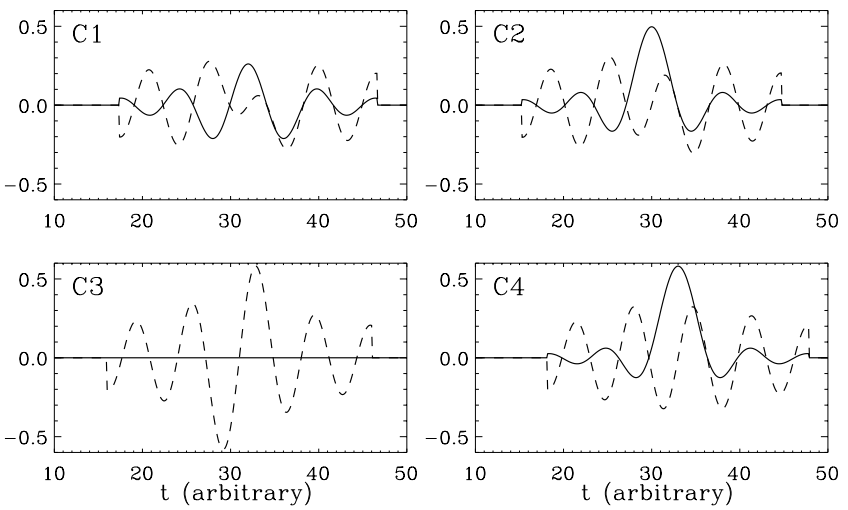

Figure B3. The same as Figure B1, but the signal of C3 is measured along the trajectory that cross the current tube center.

of time separations $\Delta t_{i j}$ that poses a lot of problems. Therefore the limits of application of the timing method are mainly associated with $\Delta t_{i j}$ determination.

[79] We determine $\Delta t_{i j}$ using the maximum of the crosscorrelation functions, see expression (13). Figure B2 shows the six functions $\mathcal{R}_{i j}(\Delta t)$ for the six satellite pairs. The maximums of the functions are well defined, and the time lags corresponding to these maxima give $\Delta t_{i j}$. The condition (12) is verified for all satellite triples and the timing method (14) gives $\mathbf{V}=(1,0,0)$. Therefore we recover exactly the speed used to generate the synthetic signals.

[80] As it is visible from Figure B1, the waveforms depend on the trajectory of the satellites. It can happens that crossing the same structure, different satellites record completely different signals. Figure B3 shows the synthetic signals for the same trajectories of the satellites $\mathrm{C} 1, \mathrm{C} 2$, and $\mathrm{C} 4$ that in Figure B1. The satellite $\mathrm{C} 3$ goes through the current tube center and we note that $\delta B_{x}=0$ in this case. The six cross-correlations for these 4 signals are presented in
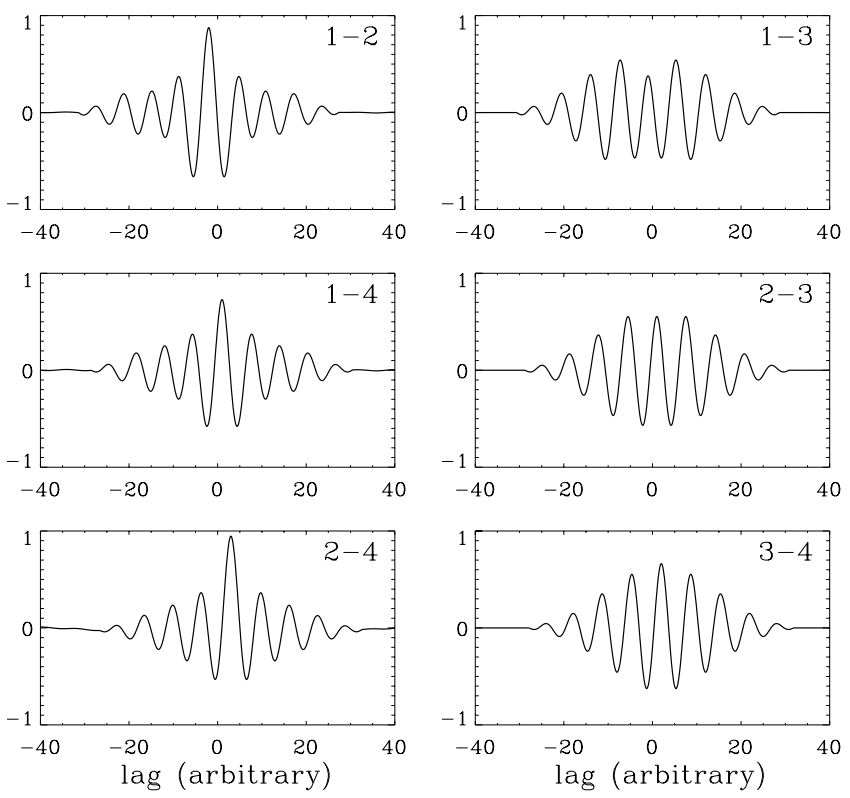

Figure B4. The same as Figure B2, but the signal of C3 is measured along the trajectory that cross the current tube center. 
Figure B4. One can see that $\mathcal{R}_{i 3}$, with $i=1,2$, do not have a well-defined maximum. In this case, the relation (12) is verified only for one satellite triplet, $\Delta t_{14}=\Delta t_{12}+\Delta t_{24}$, and the timing method (14) gives an incorrect speed $\mathbf{V}=$ $(-0.05,-0.20,0.13)$.

[81] From this numerical experience we can conclude that the timing method can be applied to determine the localized cylindrical structure speed if (1) the measured signals on the four satellites are similar; (2) the cross-correlation functions have a well-defined maximum; (3) the relation on the time separations (12) is verified for all triplets of the satellites. If one of the mentioned points is not satisfied, the results of the timing method have no meaning.

[82] Acknowledgments. We acknowledge the FGM team (PIs A. Balogh and E. Lucek) for the use of their $4 \mathrm{~s}$ resolution data. The Wavelet software was provided by C. Torrence and G. Compo and is available at http://paos.colorado.edu/research/wavelets/. O. Alexandrova thanks O. Verkhoglyadova, V. Krasnoselskih, D. Sundkvist, and A. Kropotkin for fruitful discussions and she acknowledges the support of the French Centre National d'Etude Spatiales (CNES).

[83] Zuyin Pu thanks David Burgess, William Matthaeus, and another reviewer for their assistance in evaluating this paper.

\section{References}

Alexandrova, O., A. Mangeney, M. Maksimovic, C. Lacombe, N. CornilleauWehrlin, E. A. Lucek, P. M. E. Décréau, J.-M. Bosqued, P. Travnicek, and A. N. Fazakerley (2004), Cluster observations of finite amplitude Alfvén waves and small-scale magnetic filaments downstream of a quasi-perpendicular shock, J. Geophys. Res., 109, A05207, doi:10.1029/2003JA010056.

Anderson, B. J., S. A. Fuselier, S. P. Gary, and R. E. Denton (1994), Magnetic spectral signatures in the Earth's magnetosheath and plasma depletion layer, J. Geophys. Res., 99, 5877-5891.

Balogh, A., et al. (2001), The Cluster Magnetic Field Investigation: Overview of in-flight performance and initial results, Ann. Geophys., 19, $1207-1217$.

Chmyrev, V. M., S. V. Bilichenko, V. I. Pokhotelov, V. A. Marchenko, and V. I. Lazarev (1988), Alfven vortices and related phenomena in the ionosphere and the magnetosphere, Phys. Scr., 38, 841-854.

Cornilleau-Wehrlin, N., et al. (2003), First results obtained by the Cluster STAFF experiment, Ann. Geophys., 21, 437-456.

Credland, J., G. Mecke, and J. Ellwood (1997), The Cluster Mission: ESA'S Spacefleet to the Magnetosphere, Space Sci. Rev., 79, 33-64.

Czaykowska, A., T. M. Bauer, R. A. Treumann, and W. Baumjohann (2001), Magnetic field fluctuations across the Earth's bow shock, Ann. Geophys., 19, 275-287.

Dmitruk, P., W. H. Matthaeus, and S. Oughton (2005), Direct comparisons of compressible MHD and reduced MHD turbulence, Phys. Plasmas, 12, 112,304 .

Frisch, U. (1995), Turbulence: The Legacy of A. N. Kolmogorov, Cambridge Univ. Press, New York.

Galtier, S., S. V. Nazarenko, A. C. Newell, and A. Pouquet (2002), Anisotropic turbulence of shear-Alfvén waves, Astrophys. J., 564, L49-L52, doi:10.1086/338791

Ghosh, S., and M. L. Goldstein (1997), Anisotropy in Hall MHD turbulence due to a mean magnetic field, J. Plasma Phys., 57, 129-154.

Grappin, R. (1986), Onset and decay of two-dimensional magnetohydrodynamic turbulence with velocity-magnetic field correlation, Phys. Fluids, 29, 2433-2443.

Gustafsson, G., et al. (1997), The Electric Field and Wave Experiment for the Cluster mission, Space Sci. Rev., 79, 137-156.

Hellinger, P., P. Trávníček, A. Mangeney, and R. Grappin (2003), Hybrid simulations of the magnetosheath compression: Marginal stability path, Geophys. Res. Lett., 30(18), 1959, doi:10.1029/2003GL017855.

Kadomtsev, B. B., and O. P. Pogutse (1974), Nonlinear helical perturbations of a plasma in the tokamak, Z. Eksp. Teor. Fiz., 65, 575-589.

Kevlahan, N. K.-R. (1997), The vorticity jump across a shock in a nonuniform flow, J. Fluid Mech., 341, 371-384.

Lacombe, C., and G. Belmont (1995), Waves in the Earth's magnetosheath: Observations and interpretations, Adv. Space Res., 15, 329-340.

Lacombe, C., F. G. E. Pantellini, D. Hubert, C. C. Harvey, A. Mangeney, G. Belmont, and C. T. Russell (1992), Mirror and Alfvenic waves observed by ISEE $1-2$ during crossings of the earth's bow shock Ann. Geophys., 10, 772-784.
Laveder, D., T. Passot, and P. L. Sulem (2002), Transverse dynamics of dispersive Alfvén waves. I. Direct numerical evidence of filamentation, Phys. Plasmas, 9, 293-304.

Leamon, R. J., C. W. Smith, N. F. Ness, W. H. Matthaeus, and H. K. Wong (1998), Observational constraints on the dynamics of the interplanetary magnetic field dissipation range, J. Geophys. Res., 103, 4775.

Lucek, E. A., M. W. Dunlop, T. S. Horbury, A. Balogh, P. Brown, P. Cargill, C. Carr, K.-H. Fornaçon, E. Georgescu, and T. Oddy (2001), Cluster magnetic field observations in the magnetosheath: Four-point measurements of mirror structures, Ann. Geophys., 19, 1421-1428.

Maksimovic, M., S. D. Bale, T. S. Horbury, and M. André (2003), Bow shock motions observed with CLUSTER, Geophys. Res. Lett., 30(7), 1393, doi:10.1029/2002GL016761.

Matthaeus, W. H., M. L. Goldstein, and D. A. Roberts (1990), Evidence for the presence of quasi-two-dimensional nearly incompressible fluctuations in the solar wind, J. Geophys. Res., 95, 20,673-20,683.

Matthaeus, W. H., S. Ghosh, S. Oughton, and D. A. Roberts (1996), Anisotropic three-dimensional MHD turbulence, 101, 7619-7630.

Müller, W.-C., and R. Grappin (2005), Spectral energy dynamics in magnetohydrodynamic turbulence, Phys. Rev. Lett., 95(11), 114502, doi:10.1103/PhysRevLett.95.114502.

Passot, T., and P. L. Sulem (2003), Filamentation instability of long Alfvén waves in warm collisionless plasmas, Phys. Plasmas, 10, 3914.

Petviashvili, V. I., and O. A. Pokhotelov (1992), Solitary Waves in Plasmas and in the Atmosphere, Gordon and Breach, New York.

Rème, H., et al. (2001), First multispacecraft ion measurements in and near the Earth's magnetosphere with the identical Cluster ion spectrometry (CIS) experiment, Ann. Geophys., 19, 1303-1354.

Rezeau, L., A. Roux, and C. T. Russell (1993), Characterization of smallscale structures at the magnetopause from ISEE measurements, $J$. Geophys. Res., 98, 179-186.

Rezeau, L., G. Belmont, N. Cornilleau-Wehrlin, F. Reberac, and C. Briand (1999), Spectral law and polarization properties of the low-frequency waves at the magnetopause, Geophys. Res. Lett., 26, 651-654.

Sahraoui, F., et al. (2003), ULF wave identification in the magnetosheath: The k-filtering technique applied to Cluster II data, J. Geophys. Res. 108(A9), 1335, doi:10.1029/2002JA009587.

Sahraoui, F., G. Belmont, L. Rezeau, N. Cornilleau-Wehrlin, J. L. Pinon, and A. Balogh (2006), Anisotropic turbulent spectra in the terrestrial magnetosheath as seen by the Cluster spacecraft, Phys. Rev. Lett., 96, 075,002.

Schwartz, S. J. (1998), Shock and discontinuity normals, mach numbers, and related parameters, in Analysis Methods for Multi-Spacecraft Data, ISSI Sci. Rep., pp. 249-270, ESA Publ. Div., Noordjwick, Netherlands.

Schwartz, S. J., D. Burgess, and J. J. Moses (1996), Low-frequency waves in the Earth magnetosheath: Present status, Ann. Geophys., 14, 1134-1150.

Shebalin, J. V., W. H. Matthaeus, and D. Montgomery (1983), Anisotropy in MHD turbulence due to a mean magnetic field, J. Plasma Phys., 29, $525-547$

Shukla, P. K., M. Y. Yu, and R. K. Varma (1985), Drift-Alfvén vortices, Phys. Fluids, 28, 719-721.

Sonnerup, B., and M. Scheible (1998), Minimum and maximum variance analysis, in Analysis Methods for Multi-Spacecraft Data, ISSI Sci. Rep., pp. 185-220, ESA Publ. Div., Noordjwick, Netherlands.

Strauss, H. R. (1976), Nonlinear, three-dimensional magnetohydrodynamics of noncircular tokamaks, Phys. Fluids, 19, 134-140.

Sundkvist, D., V. Krasnoselskikh, P. K. Shukla, A. Vaivads, M. André, S. Buchert, and H. Rème (2005), In situ multi-satellite detection of coherent vortices as a manifestation of Alfvénic turbulence, Nature, 436, 825-828, doi:10.1038/nature03931.

Ting, A. C., W. H. Matthaeus, and D. Montgomery (1986), Turbulent relaxation processes in magnetohydrodynamics, Phys. Fluids, 29, $3261-3274$.

Torrence, C., and G. P. Compo (1998), A practical guide to wavelet analysis, Bull. Am. Meteorol. Soc., 79, 61-78.

O. Alexandrova, A. Mangeney, and M. Maksimovic, Observatoire de Paris, Laboratoire d'Etudes Spatiales et d'Instrumentation en Astrophysique, Centre National de la Recherche Scientifique, 5 pl. J. Janssen, F-92195 Meudon, France. (olga.alexandrova@obspm.fr; mangeney@despace. obspm.fr; milan.maksimovic@obspm.fr)

M. André, Swedish Institute of Space Physics, Umeiå University, Umeiå, Sweden. (ma@irfu.se)

J.-M. Bosqued, Centre d'Etude Spatiale des Rayonnements, Centre National de la Recherche Scientifique, 9, avenue du Colonel Roche, BP 4346 F-31028 Toulouse, France. (jean-michel.bosqued@cesr.fr)

N. Cornilleau-Wehrlin, Centre d'Etude des Environnements Terrestre et Plantaires, L'Institut Pierre-Simon Laplace, 10 - 12, av. de l'Europe, F-78140 Vélizy, France. (nicole.cornilleau@cetp.ipsl.fr) 